\title{
Multisensor Management Method for Ground Moving Target Tracking Based on Doppler Blind Zone Information
}

\author{
Yunpu Zhang $\mathbb{D},{ }^{1}$ Ganlin Shan $\mathbb{D},{ }^{1}$ Rui Zhang $\mathbb{D}^{1},{ }^{1}$ and Xiusheng Duan $\mathbb{D}^{2}$ \\ ${ }^{1}$ Shijiazhuang Campus, Army Engineering University, Shijiazhuang 050003, China \\ ${ }^{2}$ Department of Mechanical Engineering, Shijiazhuang Tiedao University, Shijiazhuang 050003, China \\ Correspondence should be addressed to Yunpu Zhang; zypsensor@163.com
}

Received 23 September 2021; Revised 9 December 2021; Accepted 20 January 2022; Published 3 February 2022

Academic Editor: Chun-xi Yang

Copyright (C) 2022 Yunpu Zhang et al. This is an open access article distributed under the Creative Commons Attribution License, which permits unrestricted use, distribution, and reproduction in any medium, provided the original work is properly cited.

\begin{abstract}
Tracking ground moving target with sensors proves to be a challenge due to the uncertainty of target motion area, the existence of Doppler blind zone (DBZ), and the complex terrain. In this paper, a multisensor management method based on DBZ information is presented, in which the available sensors are selected to obtain the best operational revenues for ground moving target tracking. First, the ground target motion model is established considering the off-road/on-road state based on road topology information. Second, the sensor measurement model is given combined with the DBZ information, and a decorrelation method of measurement noise is proposed. Third, a target state estimation algorithm is derived using particle filter, in which the DBZ information is regarded as prior information. Then, combined with the variable structure interacting multiple model method, an estimation algorithm for tracking maneuvering target is proposed. Furthermore, an optimization model of nonmyopic sensor management is constructed to obtain the best sensor management scheme. Finally, the advancement and effectiveness of the proposed management method are verified in the simulations.
\end{abstract}

\section{Introduction}

With the development of sensing technology and information fusion technology, multisensor systems have been widely used in the military field [1-5]. How to determine a reasonable and effective sensor management method to obtain the best operational revenues has become a research hotspot for many scholars.

In terms of decision-making methods in sensor management, there are two kinds of methods, including the myopic sensor management method [6] and the nonmyopic sensor management method [7]. The myopic method decides the management scheme based on predicting the one-step revenue in the future. On the contrast, the nonmyopic method decides the management scheme based on predicting the multistep revenues, which can obtain a better management performance than the myopic method, but with a large computing time.

At present, scholars usually focus their research on the sensor management method based on the optimization indicator, whose connotation is to set an objective function closely related to the optimization indicator to maximize the desired operational revenues $[8,9]$. According to the selected optimization indicator, the sensor management methods can be divided into three main categories: information-indicator-based management methods, riskindicator-based management methods, and task-indicatorbased management methods. The information-indicatorbased methods focus on managing sensors to maximize the information gain, thus reducing the uncertainty in the observation. Commonly used information indicators are the Shannon entropy $[10,11]$, the Kullback-Leibler divergence [12], and the Rényi information divergence [13, 14]. However, the disadvantage of the methods is that the meaning of the used information indicators is too abstract to describe their concrete physical meaning, which may make it difficult for commanders to understand their connotation accurately. The risk-indicator-based management methods are mainly used in scenarios where sensor resources are scarce. The methods consider that the sensor management is performed to reduce the losses due to measurement uncertainty, rather than to maximize measurement performance. Commonly 
used risk indicators include the threat assessment risk [15], the target loss risk $[16,17]$, and the sensor radiation risk [18-20]. The task-indicator-based management methods focus on the combat task, and the relevant indicators are directly related to the tasks. Unlike the risk-indicator-based method, the methods are applicable when the sensor resources are redundant. Typical indicators are the covariance matrix of target state $[21,22]$, the target detection probability [23], and the posterior Cramér-Rao lower bound (PCRLB) [24, 25]. Among the three kinds of sensor management methods mentioned above, methods for target tracking have been most studied, and the representative work is shown in [19, 25, 26]. In [19], a nonmyopic sensor management method is applied to track aerial targets for the tradeoff between the tracking accuracy and the radiation risk. Mohammadi and Asif [25] present a dynamic sensor scheduling for tracking problems in distributed sensor networks, in which the PCRLB is used to quantify the tracking accuracy. Gostar et al. [26] present a constrained sensor management method for the multitarget tracking based on information divergence, and the labeled multi-Bernoulli filter was proposed for estimating the target state.

However, most of the existing studies about sensor management methods mainly focus on aerial targets and neglect ground targets. In the actual battlefield, there is inevitably a need for tracking ground targets, whose motions are uncertain and often subject to the terrain information [27-29]. Inground target tracking, the most widely used terrain information, is the road information, and the representative work is shown in [30-35]. In [30], an on-road target tracking is proposed, in which the linear roads are mapped to the ground coordinate system and the road network is established as a one-dimensional linear mode. Koch et al. [31] propose a road map extraction method to reduce the error of the road map. In [32], the road network is modeled as constant curvature segments, and the nonlinear roadconstrained Kalman filtering is used in target state estimation. In [33], the widths of road segments are considered in the state model of road target which influences the transition of the target state. Zheng and Gao [34] introduce the Gaussian mixture probability hypothesis density filter in the ground target tracking and proposed a multitarget tracking method in clutter combined with the road constraints. In [35], a ground moving target indication (GMTI) tracking problem is presented, and a variable structure interacting multiple model (VSIMM) particle filter was applied in the switching problem of road segments.

Meanwhile, the ground target detection sensors, such as the GMTI radar, mostly used the pulsed Doppler techniques to reduce the effect of clutters [36]. However, the Doppler blind zone (DBZ) problem has become a great challenge in target tracking. The low-Doppler targets, whose Doppler magnitude falls (i.e., the radial velocity of the target) below the minimum detectable velocity (MDV), cannot be detected by the sensor [37]. This results in a significant decrease in tracking accuracy. For the target tracking in the presence of the DBZ, most previous works focus on improving the filter and data fusion algorithms [38-40], but few works utilize sensor management to solve it.
To solve the problem mentioned above, we propose a multisensor management method for ground moving target tracking based on the DBZ information. The study focuses on the following points.

First, a more realistic ground moving target tracking scenario is considered in which the target can move in different motion areas and its movement is constrained by road topology information. That is to say, the target motion state is divided into two categories: off-road and on-road.

Second, a sensor measurement model under measurement uncertainty is presented considering the DBZ information. Meanwhile, Lei and Han in [41] indicate that the measurement noise of the range and the radial velocity are statistically correlated. To solve this problem, we propose a decorrelation method of measurement noise.

Third, combined with the particle filter (PF) algorithm, a target state estimation algorithm based on the DBZ information is proposed, in which the DBZ is regarded as prior information. Then, a VSIMM-PF-DBZ algorithm is proposed for tracking the maneuvering target combining the VSIMM method.

Finally, we introduce the PCRLB to quantify the tracking accuracy in the future and establish a nonmyopic sensor management optimization model to obtain the optimal management scheme.

The research framework of this paper is as follows. Section 2 describes the sensor management problem. The ground target motion model and the sensor measurement model are established in Section 3 and Section 4, respectively. In Section 5, the target state estimation algorithm based on the DBZ information is proposed. In Section 6, the VSIMM-PF-DBZ algorithm is presented. The sensor management optimization model is given in Section 7. Section 8 makes some simulations to illustrate the effectiveness and advancement of the proposed management method. Finally, conclusions are given in Section 9.

\section{Problem Description}

Sensors are Doppler radars in this paper. The target tracking scene is shown in Figure 1. Assume that there areNsensors to track a ground moving target, which can move on the road or off-road. The data processing center unifies the measurements obtained by the sensors, searches the sensor management scheme with the best revenues, and sends the corresponding commands to control the working of the sensor system. Note that we use the nonmyopic sensor management method in which the management scheme is decided based on the cumulative revenues over a time horizon in the future $[7,42]$.

At time $k$, the sensor management scheme is denoted as $A_{k}=\left(a_{k}^{n}\right)_{N \times 1}$, where $a_{k}^{n}$ indicates the working state of sensor $n$. If sensor $n$ is activated to track the target at time $k$, then $a_{k}^{n}=1$; otherwise, $a_{k}^{n}=0$. Then, the management scheme sequence over the $H$-step time horizon is denoted as $A_{k: k+H-1}=\left[A_{k}, A_{k+1}, \cdots, A_{k+H-1}\right]$.

For the convenience of presentation, we set the following two constraints related to $\mathbf{A}_{k: k+H-1}$ : 


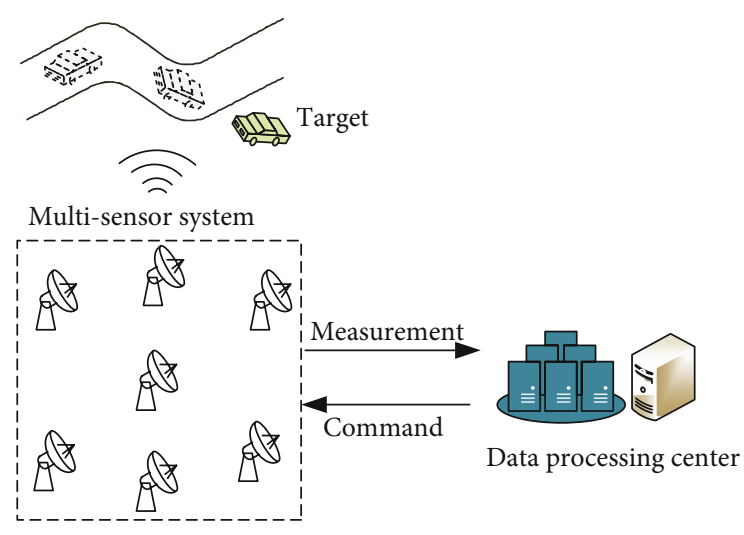

FIGURE 1: Target tracking scene.

(1) One target must be tracked by only one sensor at each time

(2) Due to the sensors cannot be switched frequently in practice, we consider the minimum dwell time $t_{\min }$ in sensor selecting. Before the working sensor can be switched, its continuous working time must exceed $t_{\min }$

The above constraints can be written as

$$
\left\{\begin{array}{l}
\sum_{n=1}^{N} a_{k+h-1}^{n}=1,1 \leq h \leq H \\
t\left(A_{k+h-1}\right) \geq t_{\min }
\end{array},\right.
$$

where $t\left(A_{k+h-1}\right)$ represents the continuous working time of the switched sensor at time $k+h-1$.

\section{Ground Target Motion Model}

The ground target state at time $k$ is $X_{k}=\left[x_{k}, \dot{x}_{k}, y_{k}, \dot{y}_{k}\right]^{\mathrm{T}}$, where $x_{k}$ and $y_{k}$ are the position coordinates in the realworld coordinate system and $\dot{x}_{k}$ and $\dot{y}_{k}$ are the corresponding velocities. When a target moves on the road, its motion state is mainly affected by the road topology [43]. For the off-road target, its motion is relatively free. Therefore, different state models are required to describe the characteristics of the target motion in different areas.

3.1. Off-Road State Model. The off-road state model can be described as [19]

$$
X_{k}=F_{i}^{\text {off-road }} X_{k-1}+G_{i}^{\text {off-road }} \boldsymbol{\varepsilon}_{k}^{\text {off-road }}
$$

where $F_{i}^{\text {off-road }}$ is the state transition matrix of motion model $i, G_{i}^{\text {off-road }}$ is the corresponding process noise gain matrix, $\boldsymbol{\varepsilon}_{k}^{\text {off-road }}$ is the zero means Gaussian process noise with covariance matrix $Q_{k}^{\text {off-road }}=\operatorname{diag}\left(\sigma_{\mathrm{x}}, \sigma_{y}\right)$, and $\tau$ is the sampling interval. In this paper, two typical motion models are considered, including the constant velocity (CV) model and the constant turn (CT) model. Thus, the corresponding state transition matrixes and process noise gain matrices are as follows [24].

$$
\begin{aligned}
& F_{\mathrm{CV}}^{\text {off-road }}=\left[\begin{array}{cccc}
1 & \tau & 0 & 0 \\
0 & 1 & 0 & 0 \\
0 & 0 & 1 & \tau \\
0 & 0 & 0 & 1
\end{array}\right] \\
& F_{C T}^{\text {off-road }}=\left[\begin{array}{cccc}
1 & \sin \varphi \pi / \varphi & 0 & -(1-\cos \varphi \pi) / \varphi \\
0 & \cos \varphi \pi & 0 & -\sin \varphi \pi \\
0 & (1-\cos \varphi \pi) / \varphi & 1 & \sin \varphi \pi / \varphi \\
0 & \sin \varphi \pi & 0 & \cos \varphi \pi
\end{array}\right], \\
& G_{\mathrm{CV}}^{\text {off-road }}=G_{\mathrm{CT}}^{\mathrm{off}-\mathrm{road}}=\left[\begin{array}{cc}
\tau^{2} / 2 & 0 \\
\tau & 0 \\
0 & \tau^{2} / 2 \\
0 & \tau
\end{array}\right]
\end{aligned}
$$

where $\varphi$ is the turn rate of the target.

3.2. On-Road State Model. The information of road segments can be collected from geographic information systems (GIS) [43]. Then, the mathematical model of the road network is established by using relevant information to represent the road network as a connection of many road segments, as shown in Figure 2. When the target is moving continuously on the road, it can be considered as moving along the road centerline without deviating largely normal to it.

When the target is moving along the centerline of road segment $l$ whose start point is $\left(x_{l}^{\text {start }}, y_{l}^{\text {start }}\right)$ and end point is $\left(x_{l}^{\text {end }}, y_{l}^{\text {end }}\right)$, its state constraint can be described as

$$
\left\{\begin{array}{l}
a_{l} x_{k}+b_{l} y_{k}+c_{l}=0, \\
\left\langle\left[\dot{x}_{k}, \dot{y}_{k}\right]^{\mathrm{T}}, \overrightarrow{\boldsymbol{c}}\right\rangle=0,
\end{array}\right.
$$

with

$$
\left\{\begin{array}{l}
a_{l}=y_{l}^{\text {start }}-y_{l}^{\text {end }}, \\
b_{l}=x_{l}^{\text {end }}-x_{l}^{\text {start }} \\
c_{l}=\left(y_{l}^{\text {end }}-y_{l}^{\text {start }}\right) x_{l}^{\text {start }}-\left(x_{l}^{\text {end }}-x_{l}^{\text {start }}\right) y_{l}^{\text {start }},
\end{array}\right.
$$

where $\left\langle\left[\dot{x}_{k}, \dot{y}_{k}\right]^{\mathrm{T}}, \overrightarrow{\boldsymbol{c}}\right\rangle$ represents the angle between two vectors, $\overrightarrow{\boldsymbol{c}}$ is the direction vector of the road segment direction, $a_{l}, b_{l}$, and $c_{l}$ are the coefficients of the centerline equation.

Equation (6) considers the state constraint when the target moves along the centerline, but the target may slightly deviate to the centerline because of the presence of the process noise. Combined with equation (6), the target state 


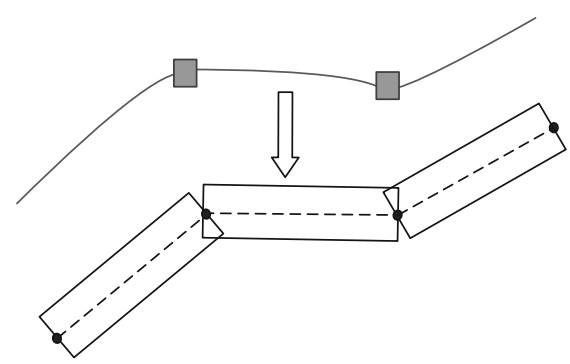

FIGURE 2: Road network modeling schematic.

constraint can be expressed as

$$
\left\{\begin{array}{l}
d\left[\left(x_{k}, y_{k}\right) \mid a_{l} x+b_{l} y+c_{l}=0\right] \leq \Delta d \\
\left\langle\left[\dot{x}_{k}, \dot{y}_{k}\right]^{\mathrm{T}}, \overrightarrow{\boldsymbol{c}}\right\rangle \leq \Delta v
\end{array},\right.
$$

where $d\left[\left(x_{k}, y_{k}\right) \mid a_{l} x+b_{l} y+c_{l}=0\right]$ represents the distance from point $\left(x_{k}, y_{k}\right)$ to line $a_{l} x+b_{l} y+c_{l}=0$ and $\Delta d$ and $\Delta v$ are the deviation threshold of the distance and velocity angle, respectively.

While satisfying the above state constraint, the on-road state model can be described as

$$
X_{k}=F_{i}^{\text {on-road }} X_{k-1}+G_{i}^{\text {on-road }} \boldsymbol{\varepsilon}_{k}^{\text {on-road }} .
$$

In this paper, we consider that the on-road target mentioned only moves in $\mathrm{CV}$ model. Therefore, $F_{i}^{\text {on-road }}=$ $F_{\mathrm{CV}}^{\text {off-road }}$, and $G_{i}^{\text {on-road }}=G_{\mathrm{CV}}^{\text {off-road }}$.

We denote the variances of the process noise along the road and orthogonal to the road as $\sigma_{\|}^{2}$ and $\sigma_{\perp}^{2}\left(\sigma_{\perp}^{2} \ll \sigma_{\|}^{2}\right)$, respectively. The covariance matrix $\mathbf{Q}_{k}^{\text {on-road }}$ after projecting it to the X-Y coordinate system can be written as [33]

$$
Q_{k}^{\text {on-road }}=\left[\begin{array}{cc}
\cos \theta_{l} & -\sin \theta_{l} \\
\sin \theta_{l} & \cos \theta_{l}
\end{array}\right]\left[\begin{array}{cc}
\sigma_{\|}^{2} & 0 \\
0 & \sigma_{\perp}^{2}
\end{array}\right]\left[\begin{array}{cc}
\cos \theta_{l} & -\sin \theta_{l} \\
\sin \theta_{l} & \cos \theta_{l}
\end{array}\right]^{\mathrm{T}},
$$

where $\theta_{l}$ represents the orientation angle of road segment $l$.

Note that the state transition of the on-road target is also determined by the road segment on which they are located, while the state transition of the off-road target is mainly determined by its motion model (CV or CT).

3.3. Motion Area Transitions. Obviously, the motion areas of the target can be switched between on-road and off-road. In this paper, parameter $m_{k}$ is used to indicate the area in which the target is moving at time $k$, where $m_{k}=0$ represents the target moving on the off-road area and $m_{k}=1$ represents the target moving on the road. The transition process of motion area can be approximated as a Markov process, and the cor- responding transition matrix can be stated as

$$
T_{k}=\left[\begin{array}{ll}
p_{00} & p_{01} \\
p_{10} & p_{11}
\end{array}\right]=\left[\begin{array}{ll}
p\left(m_{k}=0 \mid m_{k-1}=0\right) & p\left(m_{k}=1 \mid m_{k-1}=0\right) \\
p\left(m_{k}=0 \mid m_{k-1}=1\right) & p\left(m_{k}=1 \mid m_{k-1}=1\right)
\end{array}\right],
$$

with

$$
\left\{\begin{array}{l}
p\left(m_{k}=0 \mid m_{k-1}=0\right)=1-\exp \left(-\vartheta \cdot d_{k}^{\text {entry }}\right), \\
p\left(m_{k}=1 \mid m_{k-1}=0\right)=\exp \left(-\vartheta \cdot d_{k}^{\text {entry }}\right), \\
p\left(m_{k}=0 \mid m_{k-1}=1\right)=\exp \left(-\vartheta \cdot d_{k}^{\text {exit }}\right), \\
p\left(m_{k}=1 \mid m_{k-1}=1\right)=1-\exp \left(-\vartheta \cdot d_{k}^{\text {exit }}\right) .
\end{array}\right.
$$

Here, $d_{k}^{\text {entry }}$ and $d_{k}^{\text {exit }}$ represent the distance to the nearest entry point and nearest exit point of the road network, respectively. $\vartheta$ is a probability parameter (in this paper, $\vartheta=$ $0.04)$.

\section{Sensor Measurement Model}

4.1. Sensor Measurement Equation. The Doppler sensor can obtain the distance, azimuth, and radial velocity information of the target, and the corresponding measurement equation can be expressed as [19]

$$
Z_{k}^{n}=H^{n}\left(X_{k}\right)+\boldsymbol{v}^{n}=\left[\begin{array}{c}
r_{k}^{n} \\
\varphi_{k}^{n} \\
\dot{r}_{k}^{n}
\end{array}\right]+\left[\begin{array}{c}
v_{r}^{n} \\
v_{\varphi}^{n} \\
v_{\dot{r}}^{n}
\end{array}\right],
$$

with

$$
\left\{\begin{array}{l}
r_{k}^{n}=\sqrt{\left(x_{k}-x_{s}^{n}\right)^{2}+\left(y_{k}-y_{s}^{n}\right)^{2},} \\
\varphi_{k}^{n}=\arctan \left(\frac{y_{k}-y_{s}^{n}}{x_{k}-x_{s}^{n}}\right), \\
\dot{r}_{k}^{n}=\frac{\dot{x}_{k}\left(x_{k}-x_{s}^{n}\right)+\dot{y}_{k}\left(y_{k}-y_{s}^{n}\right)}{\sqrt{\left(x_{k}-x_{s}^{n}\right)^{2}+\left(y_{k}-y_{s}^{n}\right)^{2}}},
\end{array}\right.
$$

where the measurement information $\left[\begin{array}{lll}r_{k}^{n} & \varphi_{k}^{n} & \dot{r}_{k}^{n}\end{array}\right]^{\mathrm{T}}$ represent the range, azimuth, and radial velocity of the target at time $k$ and the noise $\boldsymbol{v}^{n}=\left[\begin{array}{lll}v_{r}^{n} & v_{\varphi}^{n} & v_{\dot{r}}^{n}\end{array}\right]^{\mathrm{T}}$ represent the Gaussian measurement noise with zero means. $x_{s}^{n}$ and $y_{s}^{n}$ are the position coordinates of sensor $n$.

If the target is not detected successfully, the sensor cannot obtain the measurement information. When the DBZ is not considered, the detection probability of sensor $n$ can 
be written as [44]

$\tilde{p}_{\mathrm{d}}^{n}\left(X_{k}\right)= \begin{cases}\left(p_{f}^{n}\right)^{1 /(1+S N R)}, & \text { if the target is inside line-of -sight region }, \\ 0, & \text { otherwise }\end{cases}$

with

$$
S N R=S N R_{\min }^{\mathrm{n}}\left(\frac{R_{\max }^{\mathrm{n}}}{r_{k}^{n}}\right)^{4}
$$

where $S N R$ is the signal to noise ratio and $p_{f}^{n}, S N R_{\min }^{\mathrm{n}}$, and $R_{\max }^{\mathrm{n}}$ represent the false probability, the minimum detectable SNR, and the maximum detection distance of sensor $n$, respectively.

When the DBZ is considered, the target with radial velocity below than MDV will not be detected, which is an important priori information for sensor management. The corresponding detection probability can be calculated by [37]

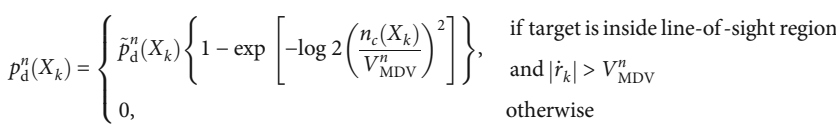

where $V_{\mathrm{MDV}}^{n}$ is the MDV of sensor $n$ and $n_{c}\left(X_{k}\right)$ is called the clutter notch function (see [37] for details).

Combined with the detection probability, the measurement equation can be written as

$$
Z_{k}^{n}=\eta H^{n}\left(X_{k}\right)+\mathbf{v}^{n}
$$

where $\eta$ represents a random number, which is taken as 0 or 1 according to the detection probability $p_{\mathrm{d}}^{n}\left(\mathbf{X}_{k}\right)$, that is

$$
p(\eta)= \begin{cases}p_{\mathrm{d}}^{n}\left(X_{k}\right), & \eta=1 \\ 1-p_{\mathrm{d}}^{n}\left(X_{k}\right), & \eta=0\end{cases}
$$

4.2. Decorrelation Method of the Measurement Noise. According to [41], the range measurement noise $v_{r}^{n}$ and the radial velocity measurement noise $v_{\dot{r}}^{n}$ are statistically correlated, which cannot be ignored in the process of target tracking. Hence, we propose a decorrelation method of measurement noise to improve the accuracy of target tracking in this paper.

We define $\sigma^{2}\left(v_{r}^{n}\right), \sigma^{2}\left(v_{\varphi}^{n}\right)$, and $\sigma^{2}\left(v_{\dot{r}}^{n}\right)$ as the variances of $v_{\varphi}^{n}, v_{\varphi}^{n}$, and $v_{\dot{r}}^{n}$, respectively. The correlation coefficient of $\sigma^{2}\left(v_{r}^{n}\right)$ and $\sigma^{2}\left(v_{\dot{r}}^{n}\right)$ is denoted as $\omega$. Then, the covariance matrix of measurement noise can be written as

$$
R^{n}=\left[\begin{array}{ccc}
\sigma^{2}\left(v_{r}^{n}\right) & 0 & \omega \sigma\left(v_{r}^{n}\right) \sigma\left(v_{\dot{r}}^{n}\right) \\
0 & \sigma^{2}\left(v_{\varphi}^{n}\right) & 0 \\
\omega \sigma\left(v_{r}^{n}\right) \sigma\left(v_{\dot{r}}^{n}\right) & 0 & \sigma^{2}\left(v_{\dot{r}}^{n}\right)
\end{array}\right] .
$$

Rewrite equation (20) as

$$
R^{n}=\left[\begin{array}{cc}
R_{r, \varphi}^{n} & \left(R_{r, \dot{r}}^{n}\right)^{\mathrm{T}} \\
R_{r, \dot{r}}^{n} & R_{\dot{r}, \dot{r}}^{n}
\end{array}\right]
$$

with

$$
\left\{\begin{array}{l}
R_{r, \varphi}^{n}=\operatorname{diag}\left[\sigma^{2}\left(v_{r}^{n}\right), \sigma^{2}\left(v_{\varphi}^{n}\right)\right] \\
R_{r, \dot{r}}^{n}=\left[\omega \sigma\left(v_{r}^{n}\right) \sigma\left(v_{\dot{r}}^{n}\right), 0\right] \\
R_{\dot{r}, \dot{r}}^{n}=\sigma^{2}\left(v_{\dot{r}}^{n}\right)
\end{array}\right.
$$

To eliminate the correlation of the elements in $R^{n}$, we use the Cholesky decomposition method and construct the decomposition matrix

$$
B^{n}=\left[\begin{array}{cc}
I_{2 \times 2} & 0 \\
L^{n} & 1
\end{array}\right]
$$

with

$$
L^{n}=-R_{r, \dot{r}}^{n}\left(R_{r, \varphi}^{n}\right)^{-1}=\left[-\omega \sigma\left(v_{\dot{r}}^{n}\right) / \sigma\left(v_{r}^{n}\right), 0\right]
$$

where $I_{2 \times 2}$ is an identity matrix.

Multiplying both sides of equation (18) simultaneously left by $B^{n}$, the decorrelated measurement equation is obtained as follows

$$
\left\{\begin{array}{l}
\tilde{Z}_{k}^{n}=\eta\left[\begin{array}{c}
r_{k}^{n} \\
\varphi_{k}^{n}
\end{array}\right]+\left[\begin{array}{c}
v_{r}^{n} \\
v_{\varphi}^{n}
\end{array}\right], \\
\tilde{\psi}_{k}^{n}=\eta \psi_{k}^{n}+v_{\psi}^{n},
\end{array}\right.
$$

where $\tilde{\psi}_{k}^{n}$ represents the pseudomeasurement of the radial velocity and $\psi_{k}$ and $v_{\psi}^{n}$ are the measurement value and noise of $\tilde{\psi}_{k}^{n}$, respectively, which can be calculated by

$$
\begin{gathered}
\psi_{k}^{n}=\frac{-\omega \sigma\left(v_{\dot{r}}^{n}\right)}{\sigma\left(v_{r}^{n}\right)} r_{k}+\dot{r}_{k}, \\
v_{\psi}^{n}=\frac{-\omega \sigma\left(v_{\dot{r}}^{n}\right)}{\sigma\left(v_{r}^{n}\right)} v_{r}^{n}+v_{\dot{r}}^{n} .
\end{gathered}
$$

Obviously, $v_{\psi}^{n}$ is the Gaussian noise with zero means, whose variance can be expressed as

$$
\sigma^{2}\left(v_{\psi}^{n}\right)=\operatorname{cov}\left(v_{\psi}^{n}, v_{\psi}^{n}\right)=\left(1-\omega^{2}\right) \sigma^{2}\left(v_{\dot{r}}^{n}\right)
$$

Therefore, the new measurement equation can be 
written as

$$
\widehat{Z}_{k}^{n}=\eta \widehat{H}^{n}\left(X_{k}\right)+\widehat{\boldsymbol{v}}^{n}=\eta\left[\begin{array}{c}
r_{k}^{n} \\
\varphi_{k}^{n} \\
\psi_{k}^{n}
\end{array}\right]+\left[\begin{array}{c}
v_{r}^{n} \\
v_{\varphi}^{n} \\
v_{\psi}^{n}
\end{array}\right] .
$$

The corresponding covariance matrix of the measurement noise is

$$
\widehat{R}^{n}=\left[\begin{array}{ccc}
\sigma^{2}\left(v_{r}^{n}\right) & 0 & 0 \\
0 & \sigma^{2}\left(v_{\varphi}^{n}\right) & 0 \\
0 & 0 & \left(1-\omega^{2}\right) \sigma^{2}\left(v_{\dot{r}}^{n}\right)
\end{array}\right] .
$$

\section{Target State Estimation Algorithm Based on the DBZ Information}

In the process of sensor management, estimating the target state at each moment is a prerequisite for system decisionmaking. When the DBZ is considered, the sensors will lose the measurements in some cases, and the target state only can be obtained by prediction based on the recurrence of the target motion models. At this time, the tracking accuracy will be greatly reduced, which is not conducive to the stable tracking of the target.

It is known that the target state in the DBZ satisfies a certain constraint relationship, that is, the radial velocity of the target is below than the MDV of the sensor, which can be used to improve the tracking performance as prior information when the sensor cannot obtain the measurements. Therefore, we propose a particle filter algorithm based on the DBZ information (PF-DBZ) for target state estimation in this paper.

For the convenience of expression, the multimodel and motion area transitions of the target are not considered in this section.

5.1. Process of the PF-DBZ. The generated particles are divided into two categories: one is the particles outside the blind zone called unconstrained particles with a number of $M_{\mathrm{UN}}$, denoted as $\left\{\mathbf{X}_{\mathrm{UN}, k}^{i}: i=1,2, \cdots, M_{\mathrm{UN}}\right\}$; another is the particles in the DBZ with a number of $M_{\mathrm{DBZ}}$, called $\mathrm{DBZ}$ particles, denoted as $\left\{\mathbf{X}_{\mathrm{DBZ}, k-1}^{i}: i=1,2, \cdots, M_{\mathrm{DBZ}}\right\}$, which are constrained by the DBZ information. At time $k$, the occurrence probability of the two kinds of particles is $p_{\mathrm{UN}, k}$ and $p_{\mathrm{DBZ}, k}$, respectively. Then, the processes of $\mathrm{PF}-\mathrm{DBZ}$ are as follows.

Step 1. State prediction.

For the unconstrained particles, the prediction state $\tilde{X}_{\mathrm{UN}, k}^{i}\left(i=1,2, \cdots, M_{\mathrm{UN}}\right)$ can be calculated based on the state model mentioned in Section 3:

$$
\tilde{X}_{\mathrm{UN}, k}^{i}=F X_{\mathrm{UN}, k-1}^{i}+G_{k} Q_{k}\left(G_{k}\right)^{\mathrm{T}} .
$$

For the DBZ particles, if $p_{\mathrm{DBZ}, k-1}>0$, the prediction state $\tilde{X}_{\mathrm{DBZ}, k}^{i}\left(i=1,2, \cdots, M_{\mathrm{DBZ}}\right)$ can also be obtained by equation (31), but the state must meet the following DBZ information constraint.

$$
\frac{\left(\dot{\tilde{x}}_{\mathrm{DBZ}, k}^{i}-\dot{x}_{s}^{n}\right)\left(\tilde{x}_{\mathrm{DBZ}, k}^{i}-x_{s}^{n}\right)+\left(\dot{\tilde{y}}_{\mathrm{DBZ}, k}^{i}-\dot{y}_{s}^{n}\right)\left(\tilde{y}_{\mathrm{DBZ}, k}^{i}-y_{s}^{n}\right)}{\sqrt{\left(\tilde{x}_{\mathrm{DBZ}, k}^{i}-x_{s}^{n}\right)^{2}+\left(\tilde{y}_{\mathrm{DBZ}, k}^{i}-y_{s}^{n}\right)^{2}}} \leq V_{\mathrm{MDV}}^{n} .
$$

Step 2. Measurement update.

At time $k$, whether sensor $n$ can obtain the measurement is an uncertain event. Therefore, different cases will be discussed as follows.

(1) Calculate the weight of the particles. The weights of the unconstrained particles $\tilde{X}_{\mathrm{UN}, k}^{i}$ can be obtained according to the likelihood function corresponding to the sensor measurement equation, that is, $\omega_{\mathrm{UN}, k}^{i}$ $\sim p\left(\widetilde{Z}_{k}^{n} \mid \tilde{X}_{\mathrm{UN}, k}^{i}\right)$. In this paper, the likelihood function is

$p\left(\widehat{Z}_{k}^{n} \mid \tilde{X}_{\mathrm{UN}, k}^{i}\right)=\frac{1}{\sqrt{2 \pi\left|\tilde{R}^{n}\right|}} \exp \left[-\frac{1}{2}\left[\widehat{Z}_{k}^{n}-\widehat{H}\left(\tilde{X}_{\mathrm{UN}, k}^{i}\right)\right]^{\mathrm{T}}\left(\widehat{R}^{n}\right)^{-1}\left[\widehat{Z}_{k}^{n}-\widehat{H}\left(\tilde{X}_{\mathrm{UN}, k}^{i}\right)\right]\right]$

(2) Normalize the weights:

$$
\omega_{\mathrm{UN}, k}^{i}=\frac{\omega_{\mathrm{UN}, k}^{i}}{\sum_{i=1}^{M_{\mathrm{UN}}} \omega_{\mathrm{UN}, k}^{i}}
$$

(3) Update the occurrence probability of two kinds of particles. Set $p_{\mathrm{UN}, k}=1$ and $p_{\mathrm{DBZ}, k}=0$

(1) Calculate the weight of the particles. Set $\omega_{\mathrm{UN}, k}^{i}=$ $\omega_{\mathrm{UN}, k-1}^{i}$ and $\omega_{\mathrm{DBZ}, k}^{i}=1 / M_{\mathrm{DBZ}}$

(2) Update the occurrence probability of two kinds of particles. Set $p_{\mathrm{UN}, k}=1-\tilde{p}_{\mathrm{d}}^{n}\left(X_{k}\right)$ and $p_{\mathrm{DBZ}, k}=\tilde{p}_{\mathrm{d}}^{n}\left(X_{k}\right)$

(1) Calculate the weight of the particles. Set $\omega_{\mathrm{UN}, k}^{i}=$ $\omega_{\mathrm{UN}, k-1}^{i}$ and $\omega_{\mathrm{DBZ}, k}^{i}=1 / M_{\mathrm{DBZ}}$

(2) Update the occurrence probability of two kinds of particles. Set $p_{\mathrm{UN}, k}=\left[1-\tilde{p}_{\mathrm{d}}^{n}\left(X_{k}\right)\right] p_{\mathrm{UN}, k-1}$ and $p_{\mathrm{DBZ}, k}$ $=1-p_{\mathrm{UN}, k}$ 
Case 1. Measurement $Z_{k}^{n}$ is existing.

Case 2. $Z_{k}^{n}$ is not existing and $p_{\mathrm{DBZ}, k-1}=0$.

Case 3. $Z_{k}^{n}$ is not existing and $p_{\mathrm{DBZ}, k-1}>0$ (continuous missing detection).
Step 3. State estimation.

The state estimation and covariance matrix after weighted average are given by

$$
\left\{\begin{array}{l}
\widehat{X}_{k}=p_{\mathrm{UN}, k} \sum_{i=1}^{M_{\mathrm{UN}}} \omega_{\mathrm{UN}, k}^{i} \tilde{X}_{\mathrm{UN}, k}^{i}++p_{\mathrm{DBZ}, k} \sum_{i=1}^{M_{\mathrm{DBZ}}} \omega_{\mathrm{DBZ}, k}^{i} \tilde{X}_{\mathrm{DBZ}, k}^{i}, \\
P_{k}=p_{\mathrm{UN}, k} \sum_{i=1}^{M_{\mathrm{UN}}} \omega_{\mathrm{UN}, k}^{i}\left(\widehat{X}_{\mathrm{UN}, k}-\tilde{X}_{\mathrm{UN}, k}^{i}\right)\left(X \wedge_{\mathrm{UN}, k}-\tilde{X}_{\mathrm{UN}, k}^{i}\right)^{\mathrm{T}}+p_{\mathrm{DBZ}, k} \sum_{i=1}^{M_{\mathrm{DBZ}}} \omega_{\mathrm{DBZ}, k}^{i}\left(\widehat{X}_{\mathrm{DBZ}, k}-\tilde{X}_{\mathrm{DBZ}, k}^{i}\right)\left(X \wedge_{\mathrm{DBZ}, k}-\tilde{X}_{\mathrm{DBZ}, k}^{i}\right)^{\mathrm{T}},
\end{array}\right.
$$

with

$$
\left\{\begin{array}{l}
\widehat{X}_{\mathrm{UN}, k}=\sum_{i=1}^{M_{\mathrm{UN}}} \omega_{\mathrm{UN}, k}^{i} \tilde{X}_{\mathrm{UN}, k}^{i} \\
\widehat{X}_{\mathrm{DBZ}, k}=\sum_{i=1}^{M_{\mathrm{DBZ}}} \omega_{\mathrm{DBZ}, k}^{i} \tilde{X}_{\mathrm{DBZ}, k}^{i}
\end{array}\right.
$$

5.2. Correction Method of Target State. According to Section 5.1 , the prediction state of the DBZ particles must meet the Doppler information constraint in the process of state prediction. For the particles that do not meet the constraint, the DBZ information can be used to correct their state to improve the accuracy of target state prediction.

For the prediction state $\tilde{X}_{\mathrm{DBZ}, k}^{i}=\left[\tilde{x}_{\mathrm{DBZ}, k}^{i}, \dot{\tilde{x}}_{\mathrm{DBZ}, k}^{i}, \tilde{y}_{\mathrm{DBZ}, k}^{i}\right.$, $\left.\dot{\tilde{y}}_{\mathrm{DBZ}, k}^{i}\right]$ of DBZ particles, when its radial velocity $\dot{\tilde{r}}_{k}^{n}>V_{\mathrm{MDV}}^{n}$ , the correction method is expressed as

$$
\left\{\begin{array}{l}
\dot{\tilde{x}}_{\mathrm{DBZ}, k}^{i}=\dot{\tilde{x}}_{\mathrm{DBZ}, k}^{i}-\left(\dot{\tilde{r}}_{k}^{n}-V_{\mathrm{MDV}}^{n}\right) \sin \left(\tilde{\varphi}_{k}^{n}\right), \\
\dot{\tilde{y}}_{\mathrm{DBZ}, k}^{i}=\dot{\tilde{y}}_{\mathrm{DBZ}, k}^{i}-\left(\dot{\tilde{r}}_{k}^{n}-V_{\mathrm{MDV}}^{n}\right) \cos \left(\tilde{\varphi}_{k}^{n}\right),
\end{array}\right.
$$

where $\tilde{\varphi}_{k}^{n}$ is the prediction value of the azimuth.

When $\dot{\tilde{r}}_{k}^{n}<-V_{\mathrm{MDV}}^{n}$, the correction method is expressed as

$$
\left\{\begin{array}{l}
\dot{\tilde{x}}_{\mathrm{DBZ}, k}^{i}=\dot{\tilde{x}}_{\mathrm{DBZ}, k}^{i}-\left(\dot{\tilde{r}}_{k}^{n}+V_{\mathrm{MDV}}^{n}\right) \sin \left(\tilde{\varphi}_{k}^{n}\right), \\
\dot{\tilde{y}}_{\mathrm{DBZ}, k}^{i}=\dot{\tilde{y}}_{\mathrm{DBZ}, k}^{i}-\left(\dot{\tilde{r}}_{k}^{n}+V_{\mathrm{MDV}}^{n}\right) \cos \left(\tilde{\varphi}_{k}^{n}\right) .
\end{array}\right.
$$

5.3. Judgment Method of Measurement Loss Causes. In ground moving target tracking, the occlusion of obstacles may also cause continuous missed detection. Therefore, it is necessary to distinguish whether the target is occluded by obstacles or enters the DBZ. According to the motion characteristics of the ground target, the radial velocity has a general tendency to decrease gradually before the target enters the DBZ. Therefore, a radial velocity sliding window is used to judge measurement loss causes in this paper.

The length of the sliding window is set to 5 , and the historical radial velocity $\left\{\dot{r}_{k-1}^{n}, \dot{r}_{k-2}^{n}, \dot{r}_{k-3}^{n}, \dot{r}_{k-4}^{n}, \dot{r}_{k-5}^{n}\right\}$ is stored in the sliding window at time $k$. Then, we define $\nabla \dot{r}_{k-i}^{n}$ as the radial velocity change rate, which is expressed as

$$
\nabla \dot{r}_{k-i}^{n}=\left(\left|\dot{r}_{k-i}^{n}\right|-\left|\dot{r}_{k-(i-1)}^{n}\right|\right) / \tau, \quad i=1,2,3,4,
$$

where $\tau$ is the sampling interval.

Using the three-fourths judgment rule, when three inequalities in equation (40) satisfy the condition, it is judged that the reason of losing measurement is entering the DBZ. In this case, the PF-DBZ and correction method of target state proposed in this paper can be used. Otherwise, it shows that the target is occluded by obstacles, and the target state only can be obtained by prediction based on the recurrence of the target motion models.

$$
\left\{\begin{array}{l}
\nabla \dot{r}_{k-1}^{n}=\left(\left|\dot{r}_{k-1}^{n}\right|-\left|\dot{r}_{k-2}^{n}\right|\right) / \tau \leq 0, \\
\nabla \dot{r}_{k-2}^{n}=\left(\left|\dot{r}_{k-2}^{n}\right|-\left|\dot{r}_{k-3}^{n}\right|\right) / \tau \leq 0, \\
\nabla \dot{r}_{k-3}^{n}=\left(\left|\dot{r}_{k-3}^{n}\right|-\left|\dot{r}_{k-4}^{n}\right|\right) / \tau \leq 0, \\
\nabla \dot{r}_{k-4}^{n}=\left(\left|\dot{r}_{k-4}^{n}\right|-\left|\dot{r}_{k-5}^{n}\right|\right) / \tau \leq 0 .
\end{array}\right.
$$

\section{VSIMM-PF-DBZ Algorithm}

The target state estimation algorithm mentioned in Section 5 is suitable for the target with a single motion model. However, according to Section 2, the motion of the ground target involves the switching of the motion model, the located road segment, and the motion area, which is difficult to describe its motion characteristics by only a single model. Hence, we combine the PF-DBZ algorithm with the VSIMM (see [45] for details) and propose a VSIMM-PF-DBZ algorithm to estimate the state of maneuvering target. In the VSIMM method, the motion model set of the target is variable at 


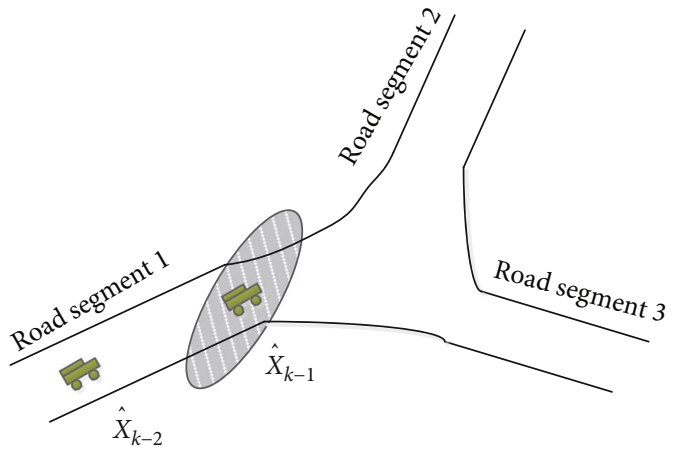

FIgURE 3: Schematic diagram of intersection.

each time, which can accurately describe the motion state of the multimodel maneuvering target.

At time $k$, the motion model set is denoted as $J_{k}=\{$ $\left.J_{k}^{\text {off-road }}, J_{k}^{\text {on-road }}\right\}$, where $J_{k}^{\text {off-road }}$ and $J_{k}^{\text {on-road }}$ represent the model set of off-road and on-road, respectively. In this paper, $J_{k}^{\text {off-road }}$ is fixed which contains CV and CT models, and $J_{k}^{\text {on-road }}$ changes in real time according to the target location. Therefore, updating $J_{k}$ means updating $J_{k}^{\text {on-road }}$.

When the target moves in the road network, its motion is mainly constrained by the road topology, so the road information can be used to assist the target estimation. Since the road information can be obtained from GIS in advance and generally does not change in target tracking, the motion model set update strategy can be designed based on the road information.

6.1. Motion Model Set Update Strategy. The motion model of the target moving on a certain road segment will generally not change, and only at intersections will the target switch the road segment and its motion model will be changed. Therefore, to update the model set of the next time, it is necessary to determine which road segment the target will be located at the next time and whether the target is close to the intersection.

As seen from Figure 3, the possible location of the target can be obtained according to the target state $\widehat{X}_{k-1}^{n}$. Then, it can be judged whether the target is close to the intersection, such as the ellipse region in Figure 3. The elliptic region can be expressed as an inequality

$$
\left[x-\widehat{x}_{k-1}, y-\widehat{y}_{k-1}\right] P_{k-1}^{\mathrm{pos}}\left[\begin{array}{l}
x-\widehat{x}_{k-1} \\
y-\widehat{y}_{k-1}
\end{array}\right] \leq \beta,
$$

where $P_{k-1}^{\text {pos }}$ is the position component of $P_{k-1}$ and $\beta$ represents the gate threshold (in this paper, $\beta=6$ ).

If intersection $i$ with coordinates $\left(x_{\text {intersection }}^{i}, y_{\text {intersection }}^{i}\right)$ satisfies inequality (41), it means that the target is close to the intersection, and the model set needs to be updated. The processes of updating $J_{k-1}^{\text {on-road }}$ are as follows.

Step 1. Get the target state $\left(\widehat{\mathbf{X}}_{k-1}, \boldsymbol{P}_{k-1}\right)$ and model set $\mathbf{J}_{k-1}^{\text {on-road }}$ at time $k$.

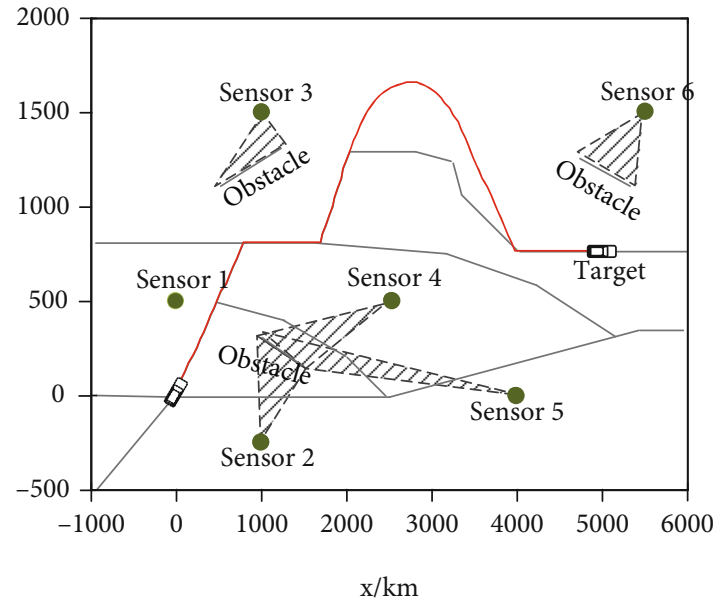

Figure 4: Schematic diagram of target tracking scene.

TABLE 1: The parameters of the sensors.

\begin{tabular}{|c|c|c|c|c|}
\hline $\begin{array}{l}\text { Sensor } \\
\text { number }\end{array}$ & Coordinates & $\begin{array}{c}\text { The } \\
\text { standard } \\
\text { deviation of } \\
\text { the range } \\
\text { noise }\end{array}$ & $\begin{array}{l}\text { The } \\
\text { standard } \\
\text { deviation of } \\
\text { the azimuth } \\
\text { noise }\end{array}$ & $\begin{array}{l}\text { The standard } \\
\text { deviations of } \\
\text { the radial } \\
\text { velocity noise }\end{array}$ \\
\hline 1 & $(0,500) \mathrm{m}$ & $40 \mathrm{~m}$ & $5 \mathrm{mrad}$ & $10 \mathrm{~m} / \mathrm{s}$ \\
\hline 2 & $\begin{array}{c}(1000,-250) \\
\mathrm{m}\end{array}$ & $20 \mathrm{~m}$ & $5 \mathrm{mrad}$ & $5 \mathrm{~m} / \mathrm{s}$ \\
\hline 3 & $\begin{array}{l}(1000, \\
1500) \mathrm{m}\end{array}$ & $25 \mathrm{~m}$ & $8 \mathrm{mrad}$ & $10 \mathrm{~m} / \mathrm{s}$ \\
\hline 4 & $\begin{array}{c}(2550,500) \\
\mathrm{m}\end{array}$ & $35 \mathrm{~m}$ & $5 \mathrm{mrad}$ & $15 \mathrm{~m} / \mathrm{s}$ \\
\hline 5 & $(4000,0) \mathrm{m}$ & $45 \mathrm{~m}$ & $5 \mathrm{mrad}$ & $25 \mathrm{~m} / \mathrm{s}$ \\
\hline 6 & $\begin{array}{c}(5500, \\
1500) \mathrm{m}\end{array}$ & $50 \mathrm{~m}$ & $7.5 \mathrm{mrad}$ & $20 \mathrm{~m} / \mathrm{s}$ \\
\hline
\end{tabular}

Step 2. Judge whether the target has closed to the intersection according to equation (41). If the target is close to the intersection, the corresponding motion models of all the links connected to the intersection are added to the model set $J_{k}^{\text {on-road}}$; otherwise, go to Step 3.

Step 3. Determine the road segment to which the target belongs at time $k$ according to $\widehat{X}_{k-1}^{n}$. If the target belongs to the road section changes, the motion model of the previous road segment is discarded and the motion model of the new road segment is added in $J_{k}^{\text {on-road; }}$ otherwise, $J_{k}^{\text {on-road }}=$ $J_{k-1}^{\text {on-road }}$.

6.2. Process of the VSIMM-PF-DBZ Algorithm. Based on the motion model set updating strategy, the VSIMM-PF-DBZ algorithm is proposed by combining the VSIMM with the PF-DBZ algorithm. Due to the uncertainty of the target motion area, we use dual filter algorithm to obtain the target state estimation of off-road state and on-road state and then apply the weighted fusion method according to motion area 
transition probability to obtain the overall state estimation. The steps of VSIMM-PF-DBZ are as follows.

\section{Step 1. Initialization.}

Obtain the target state $\left(\widehat{X}_{k-1}, P_{k-1}\right)$, the motion model set $J_{k-1}$, and the parameter $m_{k-1}$. Then, according to Section 3.3, calculate the motion area transition matrix $T_{k}$, and obtain $p\left(m_{k}=0 \mid m_{k-1}\right)$ and $p\left(m_{k}=1 \mid m_{k-1}\right)$.

Step 2. Update the motion model set.

According to the motion model set update strategy proposed in Section 6.1, obtain the model set $J_{k}^{\text {on-road }}$ of on-road state.
Step 3. State estimation.

Use the PF-DBZ algorithm in the framework of the VSIMM [45] to predict and update the target states and corresponding covariance matrices of all motion models. Then, the target state estimation of the on-road state and off-road state can be obtained, which are stated as $\left(\widehat{X}_{k}^{\text {on-road }}, P_{k}^{\text {on-road }}\right)$ and $\left(\widehat{X}_{k}^{\text {off-road }}, P_{k}^{\text {off-road }}\right)$, respectively.

Step 4. Overall estimation.

Combined with $\left(\widehat{X}_{k}^{\text {on-road }}, P_{k}^{\text {on-road }}\right),\left(\widehat{X}_{k}^{\text {off-road }}, P_{k}^{\text {off-road }}\right), p$ $\left(m_{k}=0 \mid m_{k-1}\right)$, and $p\left(m_{k}=1 \mid m_{k-1}\right)$, the overall estimation of the target state is calculated as

$$
\left\{\begin{array}{l}
\widehat{X}_{k}=p\left(m_{k}=1 \mid m_{k-1}\right) \widehat{X}_{k}^{\text {on-road }}+p\left(m_{k}=0 \mid m_{k-1}\right) \widehat{X}_{k}^{\text {off-road }} \\
P_{k}=p\left(m_{k}=1 \mid m_{k-1}\right)\left[P_{k}^{\text {on-road }}+\left(\widehat{X}_{k}^{\text {on-road }}-\widehat{X}_{k}\right)\left(X \wedge_{k}^{\text {on-road }}-X \wedge_{k}\right)^{\mathrm{T}}\right]+p\left(m_{k}=0 \mid m_{k-1}\right)\left[P_{k}^{\text {off-road }}+\left(\widehat{X}_{k}^{\text {off-road }}-\widehat{X}_{k}\right)\left(X \wedge_{k}^{\text {off-road }}-X \wedge_{k}\right)^{\mathrm{T}}\right] .
\end{array}\right.
$$

\section{Sensor Management Optimization Model}

The core of sensor management is predicting the revenue corresponding to different decision schemes in the future and selecting the schemes with the best revenue [19]. Therefore, it is important to select an optimization index for quantifying the revenue. The trace of PCRLB can predict the theoretical lower bound of the target state estimation error, which is often used to reflect the sensor tracking performance [24]. Hence, in this paper, the trace of PCRLB is used as the optimization index in the sensor management process.

According to the relevant theory of PCRLB [24], the following inequality exists.

$$
E\left[\left(\widehat{X}_{k}-X_{k}\right)\left(X \wedge_{k}-X_{k}\right)^{\mathrm{T}}\right] \geq \Psi\left(X \wedge_{k}\right)^{-1}
$$

where $\Psi\left(X \wedge_{k}\right)^{-1}$ represents the PCRLB of $\widehat{X}_{k}$, which is the inverse of the Fisher information matrix $\Psi\left(\widehat{X}_{k}\right)$.

$\Psi\left(\widehat{X}_{k}\right)$ satisfies the following recurrence relation:

$$
\Psi\left(\widehat{X}_{k}\right)=D_{k-1}^{22}-D_{k-1}^{21}\left(\Psi\left(X \wedge_{k-1}\right)+D_{k-1}^{11}\right)^{-1} D_{k-1}^{12}+D_{k}^{Z},
$$

with

$$
\left\{\begin{array}{l}
D_{k-1}^{11}=E\left[-\nabla_{\mathbf{X}_{k-1}}^{\mathbf{X}_{k-1}} \log p\left(X_{k} \mid X_{k-1}\right)\right] \\
D_{k-1}^{12}=\left[D_{k-1}^{21}\right]^{\mathrm{T}}=E\left[-\nabla_{\mathbf{X}_{k-1}}^{\mathbf{X}_{k}} \log p\left(X_{k} \mid X_{k-1}\right)\right] \\
D_{k-1}^{22}=E\left[-\nabla_{\mathbf{X}_{k}}^{\mathbf{X}_{k}} \log p\left(X_{k} \mid X_{k-1}\right)\right] \\
D_{k}^{Z}=E\left[-\nabla_{\mathbf{X}_{k}}^{\mathbf{X}_{k}} \log p\left(Z_{k} \mid X_{k}\right)\right]
\end{array}\right.
$$

where the symbol $\nabla$ represents the second-order derivative and $D_{k}^{Z}$ is the Fisher information gain. When the sensor cannot obtain the measurement, there is $D_{k}^{Z}=0$. Furthermore, for the Gaussian system discussed in this paper, there is

$$
\left\{\begin{array}{l}
D_{k-1}^{11}=\left(F_{k}\right)^{\mathrm{T}}\left(Q_{k}\right)^{-1} F_{k}, \\
D_{k-1}^{12}=\left[D_{k-1}^{21}\right]^{\mathrm{T}}=-\left(F_{k}\right)^{\mathrm{T}}\left(Q_{k}\right)^{-1} \\
D_{k-1}^{22}=\left(Q_{k}\right)^{-1} \\
D_{k}^{Z}=p_{\mathrm{d}}^{n}\left(X_{k}\right)\left[\widehat{h}^{n}\right]^{\mathrm{T}}\left(\widehat{R}^{n}\right)^{-1} \widehat{h}^{n}
\end{array}\right.
$$

where $\stackrel{\overparen{h}}{ }^{n}$ is the Jacobian matrix of measurement function $\widehat{H}^{n}\left(X_{k}\right)$ and $F_{k}$ and $Q_{k}$ represent the state transition matrix and covariance matrix of the process noise corresponding to the real target motion model at time $k$, respectively. Obviously, it is impossible to obtain the real target motion model at time $k$ in the process of decision-making, so the motion model corresponding to the maximum distribution probability at the current time is selected to do prediction [24].

In this paper, the sensor management method is nonmyopic, in which the management scheme is decided based on the cumulative revenues over a time horizon in the future. When the decision step is $H$, combining the sensor management scheme $A_{k: k+H-1}$ in the time domain $[k, k+H$ $-1]$ and the constraints equation (1), the objective 


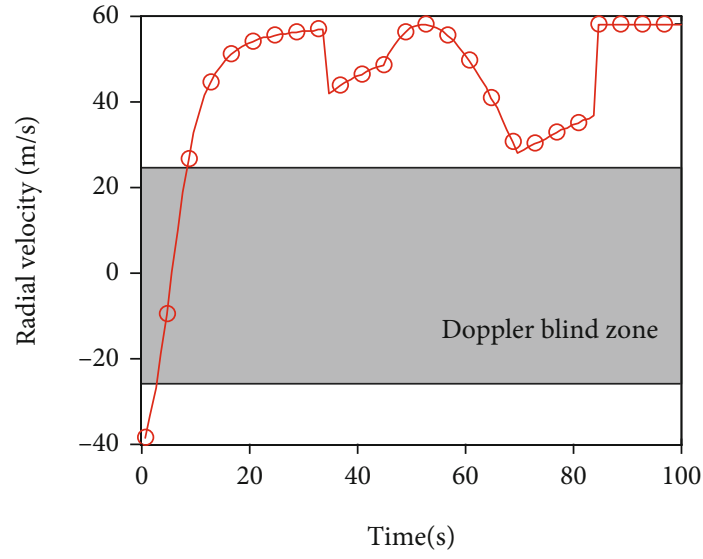

(a) Sensor 1

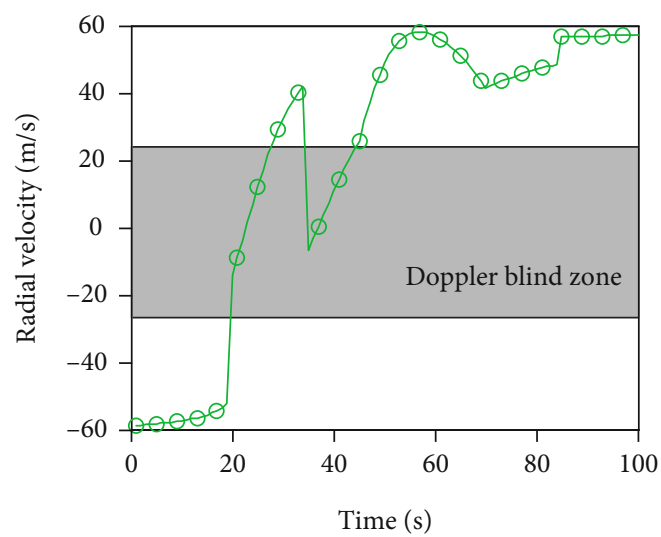

(c) Sensor 3

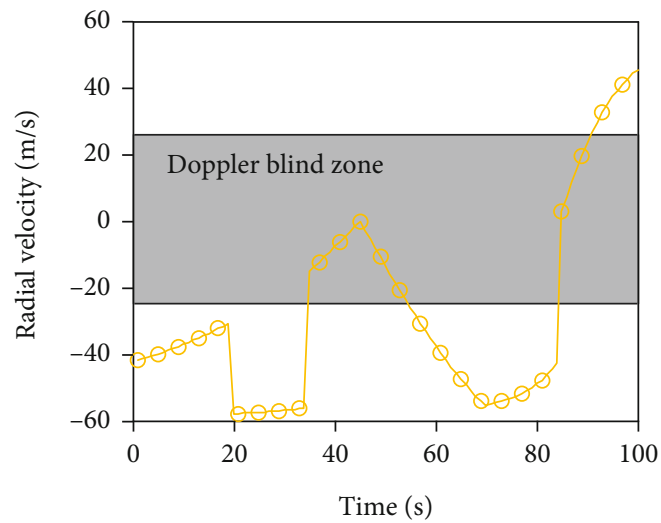

(e) Sensor 5

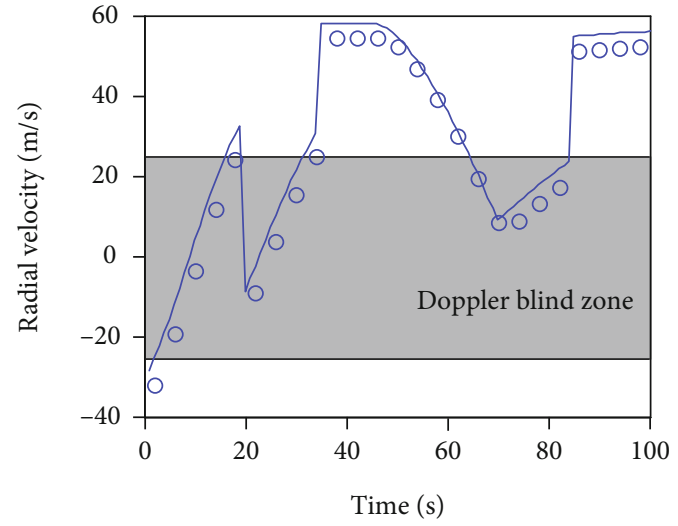

(b) Sensor 2

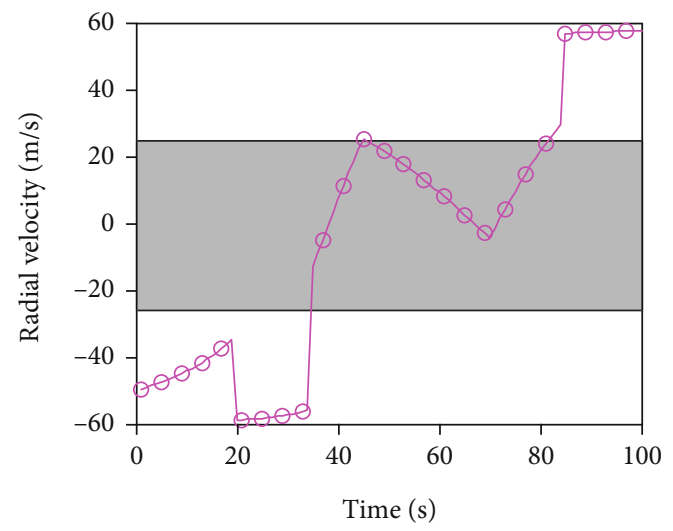

(d) Sensor 4

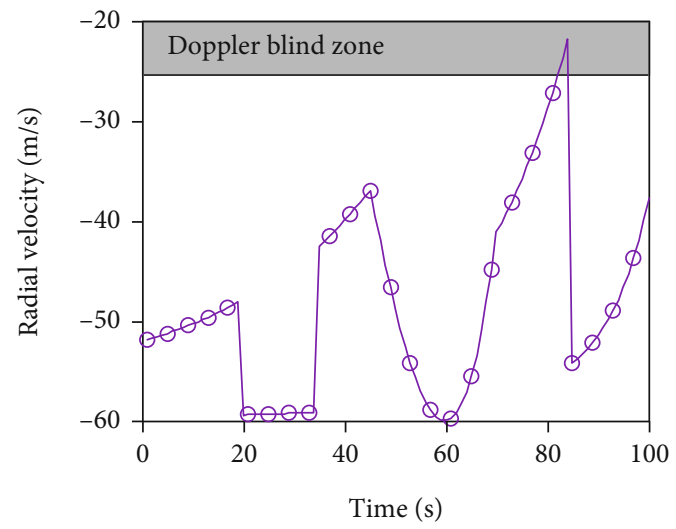

(f) Sensor 6

Figure 5: The radial velocities of the target relative to all sensors.

optimization function is established as follows.

$$
\begin{aligned}
& \min \Re\left(A_{k: k+H-1}\right)= \underbrace{\operatorname{tr}\left[\Psi\left(X \wedge_{k}, A_{k}\right)^{-1}\right]}_{\text {One-step revenue }}+\underbrace{\sum_{h=1}^{H-1} \gamma^{h} \operatorname{tr}\left[\Psi\left(X \wedge_{k+h}, A_{k+h}\right)^{-1}\right]}_{\text {Future expected revenue }}, \\
& \text { s.t. }\left\{\begin{array}{l}
\sum_{n=1}^{N} a_{k+h-1}^{n}=1,1 \leq h \leq H, \\
t\left(A_{k+h-1}\right) \geq t_{\text {min }}
\end{array}\right.
\end{aligned}
$$

where $\operatorname{tr}\left[\Psi\left(X \wedge_{k}, A_{k}\right)^{-1}\right]$ represents the trace of PCRLB after executing sensor management scheme $A_{k}$ and $\gamma(0 \leq \gamma \leq 1)$ is a discount coefficient that indicates. The optimal solution $A_{k: k+H-1}^{\mathrm{opt}}$ of the objective function is the best sensor management scheme in the time domain $[k, k+H-1]$.

\section{Numerical Simulations}

As can be seen from Figure 4, in our simulations, six Doppler sensors are used to track a ground moving target that can move on the road or off-road. Besides, there are some obstacles in the battlefield which may obstruct the detection of the 


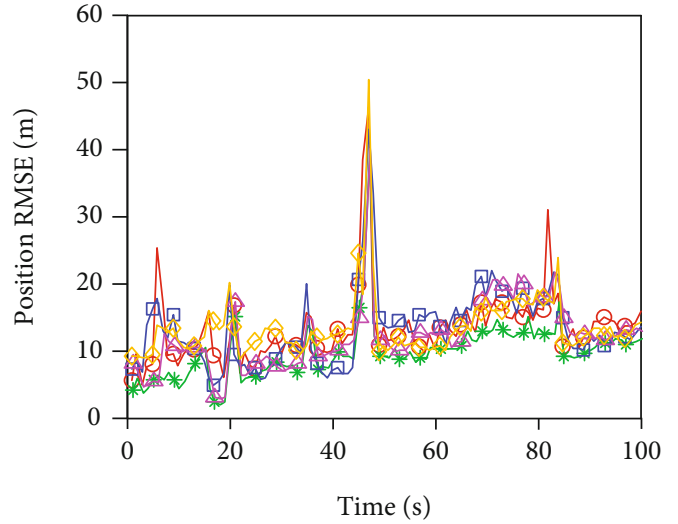

(a) Position RMSE

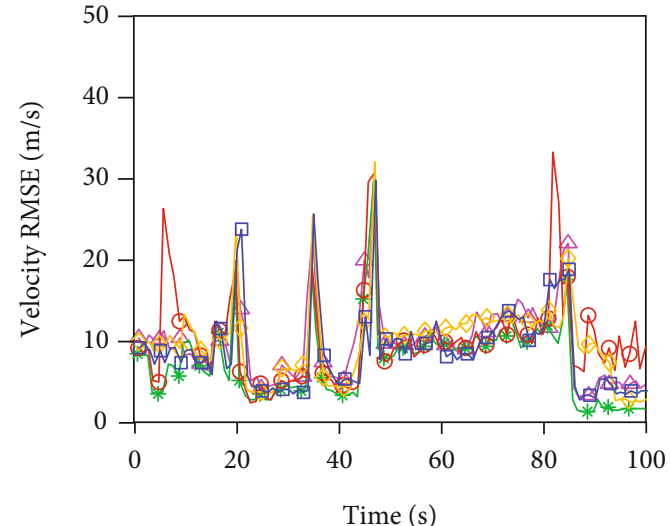

$\begin{array}{ll}\rightarrow \mathrm{H}=1 & \rightarrow \mathrm{H}=5 \\ \rightarrow \mathrm{H}=2 & \rightarrow \mathrm{H}=4 \\ \rightarrow \mathrm{H}=3 & \end{array}$

(b) Velocity RMSE

Figure 6: Comparisons of the RMSE under different decision steps.

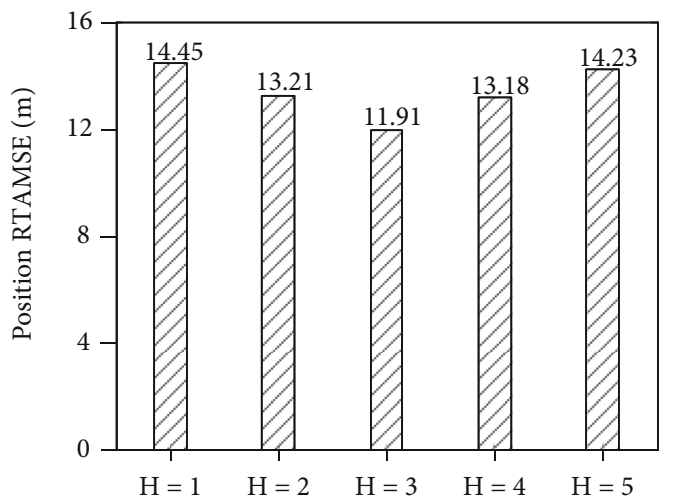

(a) Position RTAMSE

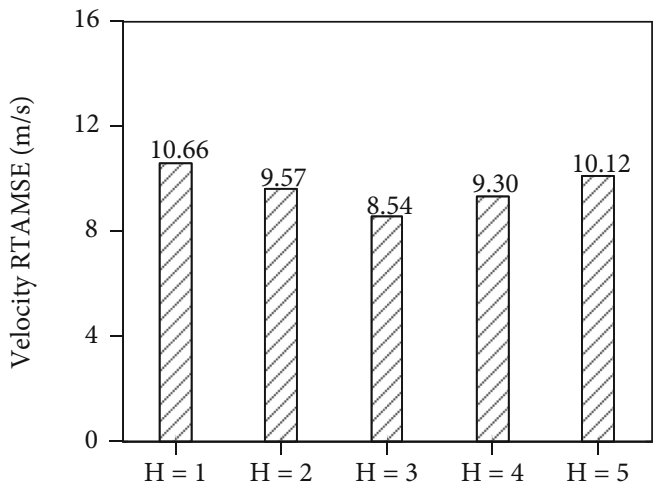

(b) Velocity RTAMSE

Figure 7: Comparison of the RTAMSE under different decision steps.

target. The sensor sampling interval is $1 \mathrm{~s}$ and the simulation duration is $100 \mathrm{~s}$.

The initial position and velocity of the target are $(0,0) \mathrm{m}$ and $60 \mathrm{~m} / \mathrm{s}$, respectively. The target moves off-road by turning right at an angle of $4^{\circ}$ during 46-70 s, moves off-road in uniform straight line during 71-85 s, and maintains uniform straight line on road during other times. The process noise variance along the $\mathrm{X}$ and $\mathrm{Y}$ directions are $20 \mathrm{~m}$ and $20 \mathrm{~m}$, and the process noise variances along the road and orthogonal to the road are $20 \mathrm{~m}$ and $1 \mathrm{~m}$.

All sensors have the same MDV $V_{\mathrm{MDV}}=25 \mathrm{~m} / \mathrm{s}$, the minimum dwell time $t_{\min }=3 \mathrm{~s}$, the false alarm probability $p_{f}=10^{-6}$, the minimum detectable $\mathrm{SNR} S N R_{\min }=15 \mathrm{db}$, and the maximum detection distance $R_{\max }=10 \mathrm{~km}$. The other parameters of the sensors are shown in Table 1.

The other parameters used in simulations are displayed as follows: the discount coefficient $\gamma=0.9$, the correlation coefficient $\omega$ of the measurement noise is 0.5 , and the number of the unconstrained and DBZ particles is 100 in the PFDBZ algorithm.
All the results were obtained by 100 trials of Monte Carlo simulations.

Figure 5 shows the radial velocities of the target relative to all sensors. Obviously, for each sensor, there is a situation where the radial velocity of the target is below than MDV, which means that the target will enter the DBZ of the sensor at some times and a single sensor cannot achieve continuous tracking of the target. Therefore, it is necessary for tracking the target to use an effective sensor management method.

8.1. Determination of the Decision Step. The decision step $H$ is a very important parameter in sensor management, which can directly affect the effectiveness of target tracking [24]. Hence, the performances of target tracking under different $H$ are studied in our simulations, and the corresponding result is used as a basis to determine the value of $H$. Figures 6 and 7 show the comparisons of the root mean square error (RMSE) and the root time average square error (RTAMSE) of the target under different $H$, respectively. 


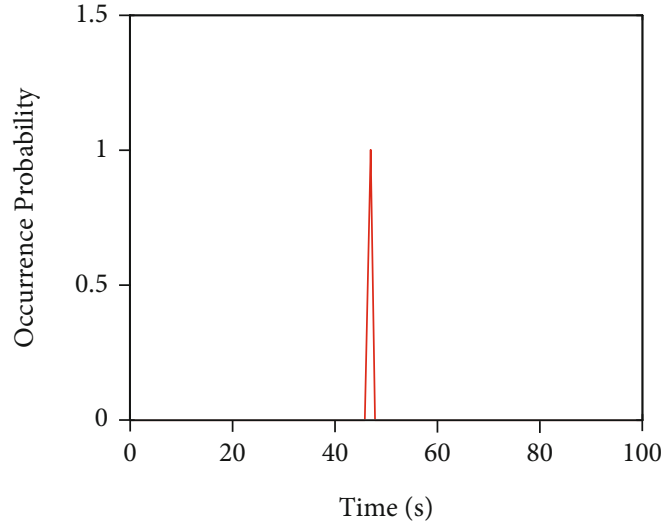

(a) PSMM

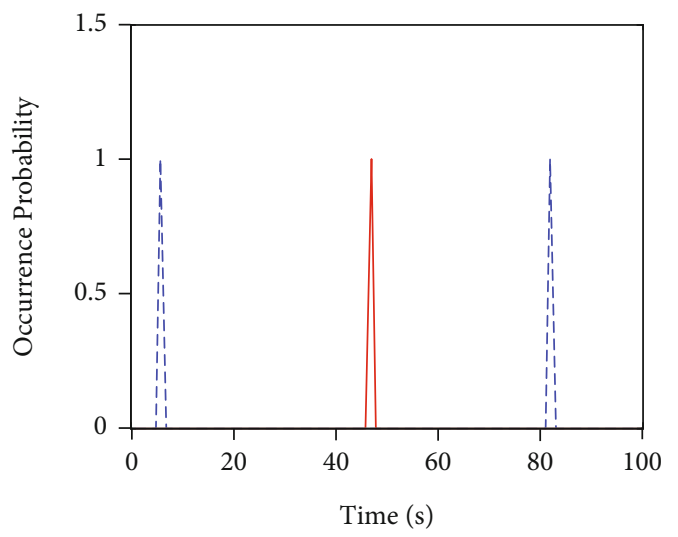

(c) CSMM

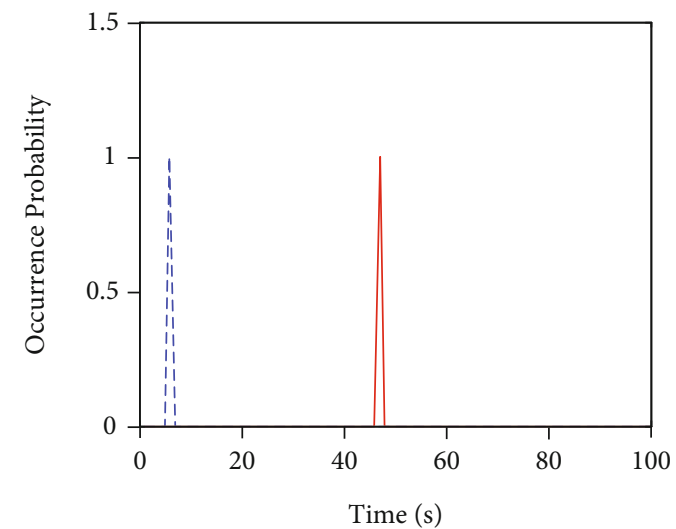

(b) MSMM

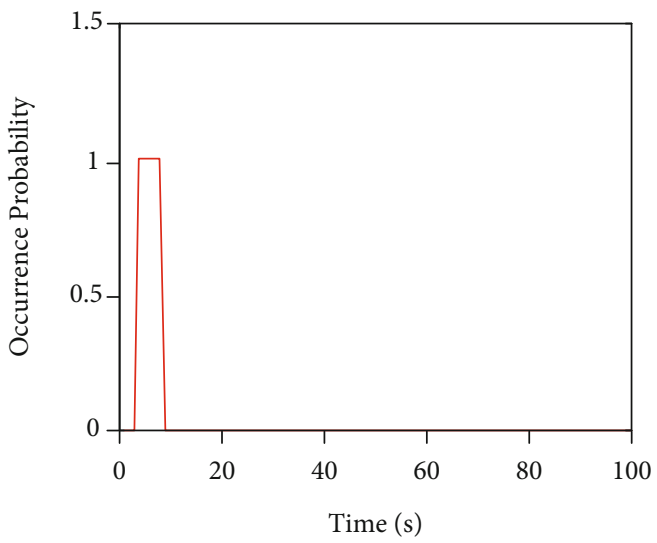

- Doppler blind zone -_- Visual blind zone

Figure 8: The occurrence probabilities of blind zones under different sensor management methods.

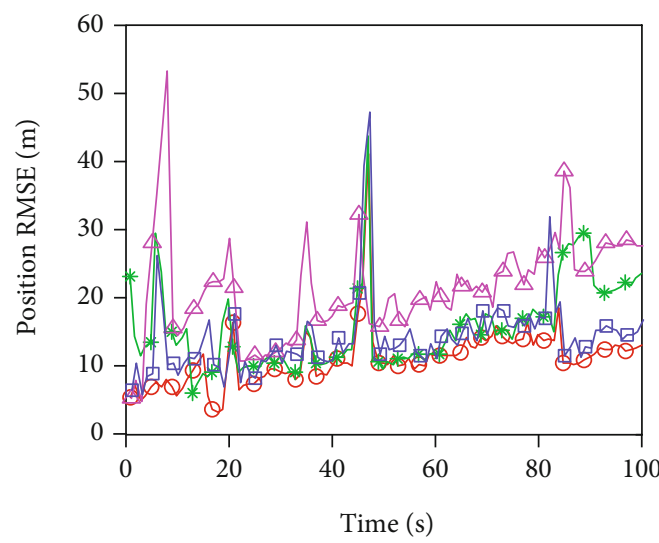

(a) Position RMSE

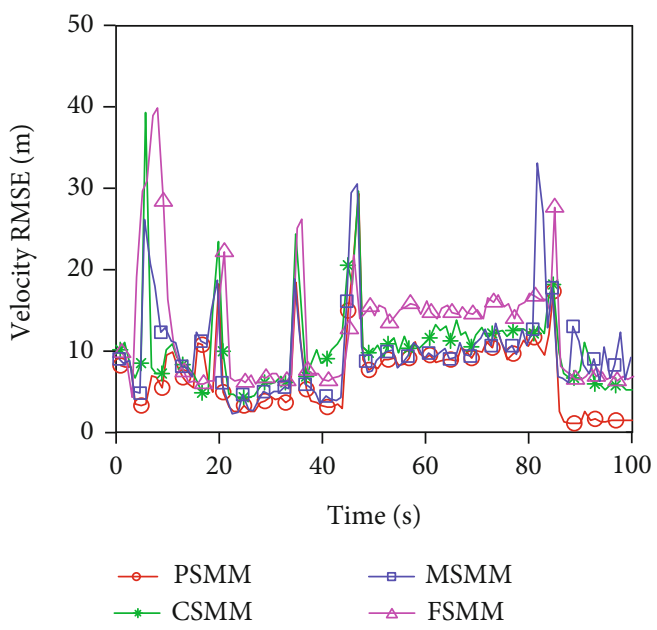

(b) Velocity RMSE

Figure 9: Comparisons of the RMSE under different sensor management methods.

As can be seen from Figures 6 and 7 , when $H=1 \sim 3$, with the increase of $H$, the RMSE and RTAMSE of the target decrease gradually, which means that the effect of tracking target is getting better and better. However, when $H>3$, the corresponding tracking errors (RMSE and RTAMSE) increase instead of decreasing with the increase of $H$. 


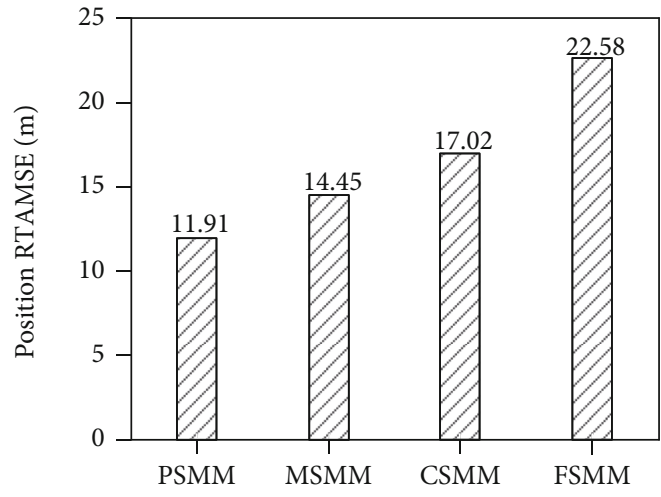

(a) Position RTAMSE

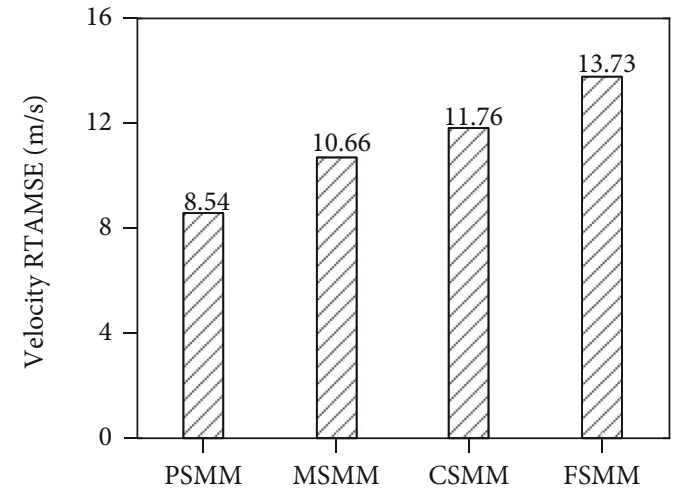

(b) Velocity RTAMSE

FIgURE 10: Comparisons of the RTAMSE of different sensor management methods.

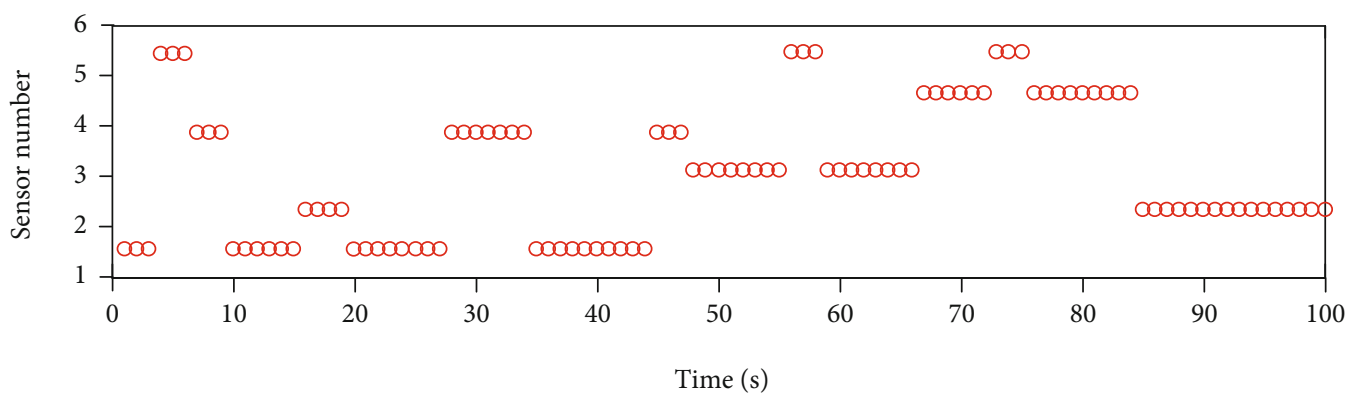

FIgURE 11: Sensor management schemes of the PSMM.

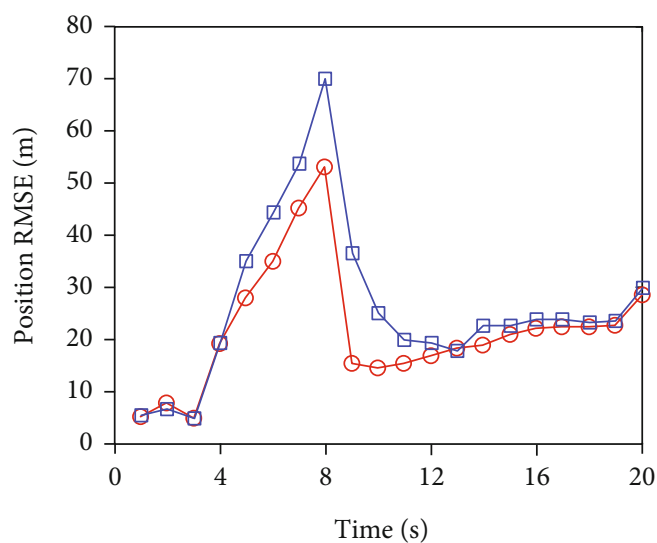

(a) Position RMSE

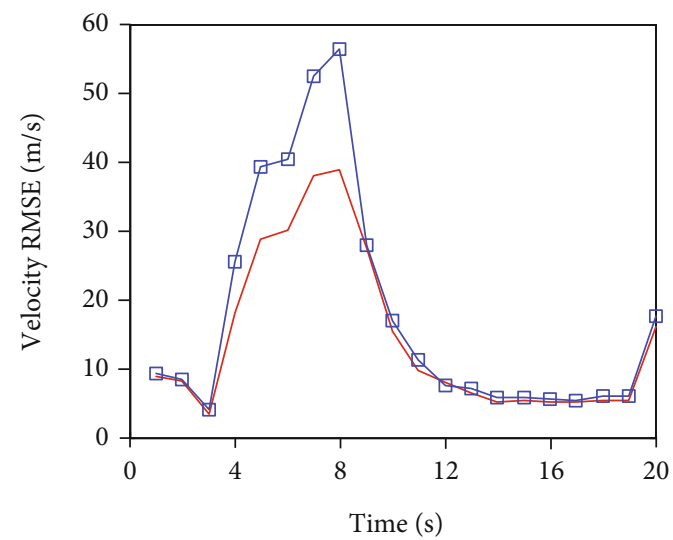

- VSIMM-PF-DBZ

$\because$ VSIMM-PF

(b) Velocity RMSE

FIgURE 12: Comparisons of the RMSE of different target state estimation algorithms.

Especially when $H=4$, the corresponding errors are larger than the errors under $H=3$. The reason for these is that with the increase of $H$, the prediction error of the target state in the future will also increase, resulting in the increase of the target tracking error. At the same time, the occurrence of measurement uncertainty events such as the target enters the DBZ which will also lead to the increase of the tracking error in the multistep prediction. Therefore, it is not the case that a larger decision step is better for tracking. Based on the above analysis, we choose $H=3$ in next simulations.

8.2. Analysis of the Proposed Sensor Management Method. In order to clearly analyze the performance of the proposed sensor management method (PSMM) in this paper, we selected three existing sensor management methods to compare with it: 


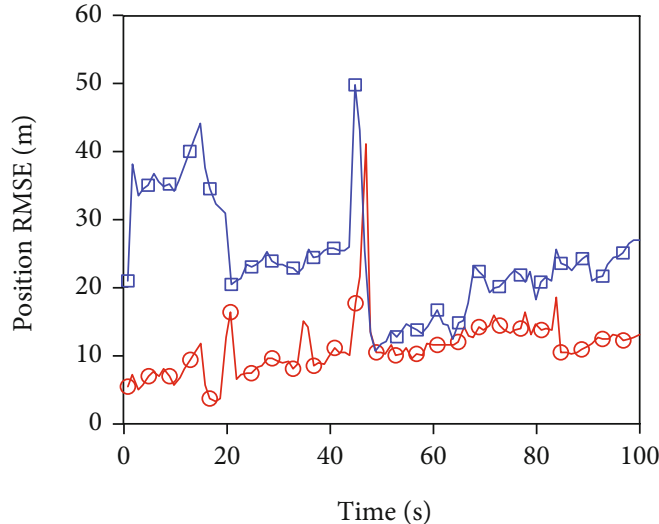

(a) Position RMSE

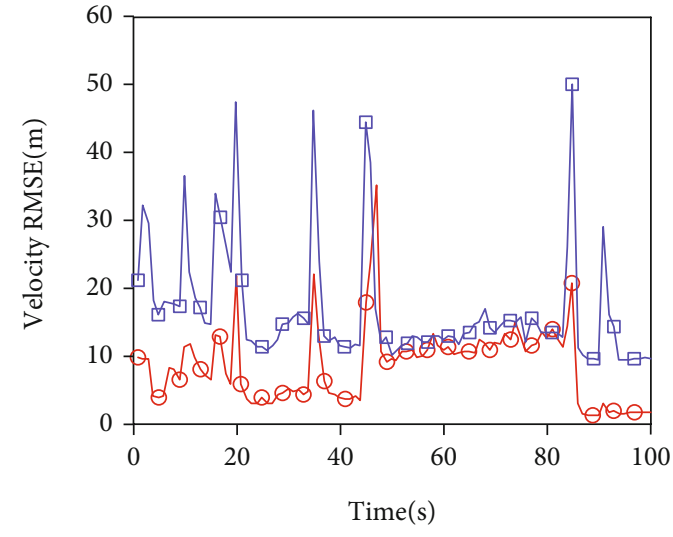

- Result with the propsed motion model $\rightarrow$ Result without road topology information (b) Velocity RMSE

FIgURE 13: Comparisons of the RMSE under different motion models.

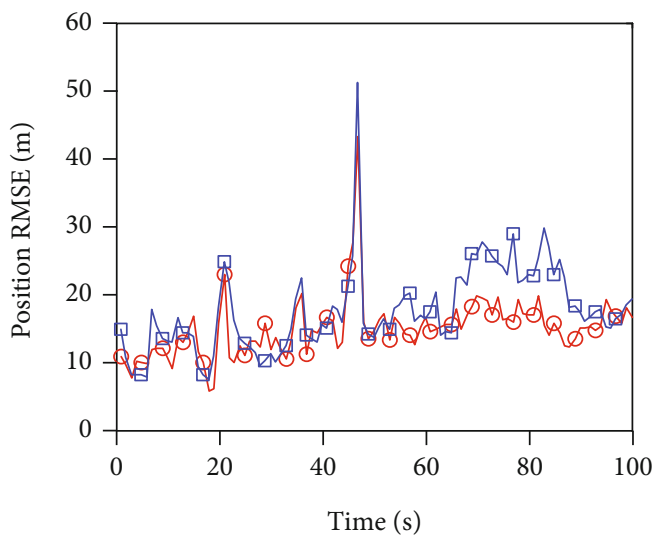

(a) Position RMSE

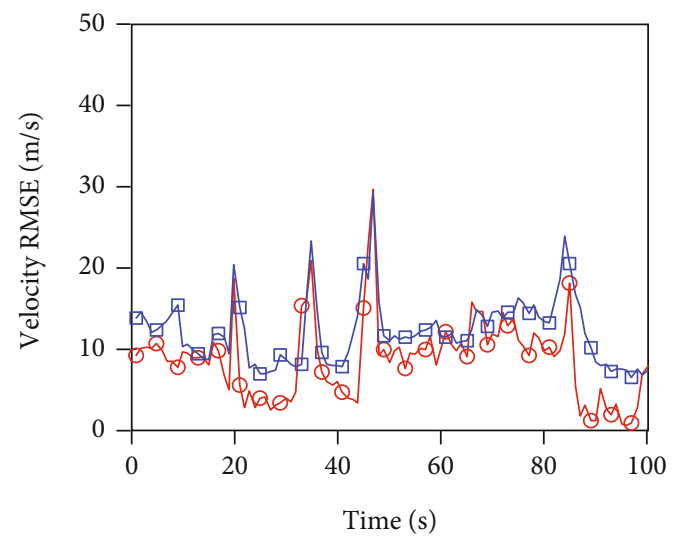

Result with the proposed decorrelation method $\square-$ Result without the decorrelation method

(b) Velocity RMSE

FIGURE 14: Comparisons of the RMSE under different measurement noise treatment methods $(\varpi=0.1)$.

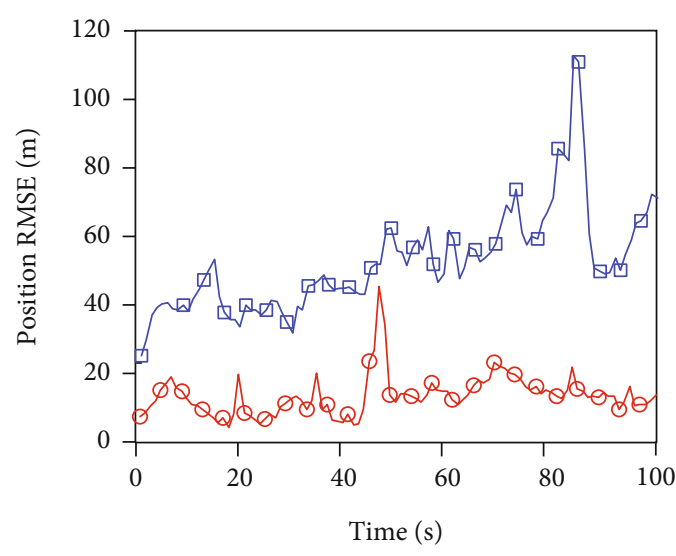

(a) Position RMSE

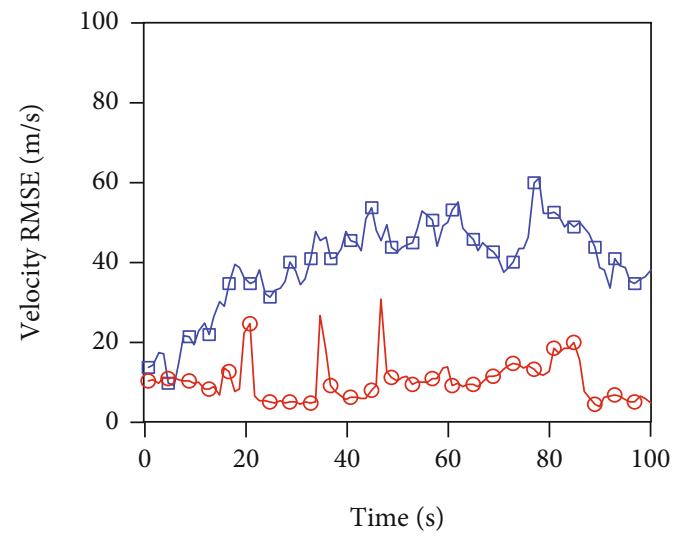

$\rightarrow$ Result with the proposed decorrelation method

$\square$ Result without the decorrelation method

(b) Velocity RMSE

Figure 15: Comparisons of the RMSE under different measurement noise treatment methods $(\omega=0.8)$. 
(1) The myopic sensor management method (MSMM), in which the management scheme is decided based on one-step revenues in the future

(2) The closest sensor management method (CSMM), in which the sensor closest to the target is selected for tracking [19]

(3) The fixed sensor management method (FSMM), in which the fixed sensor is selected for tracking in the simulation duration (in our simulations, sensor 1 is selected)

The occurrence probabilities of blind zones under different sensor management methods in 100 repeated simulations are shown in Figure 8. For the convenience of presentation, we call the target to enter the visual blind zone (VBZ) when it is blocked by obstacles. Compared with the other methods, we can see that the DBZ and VBZ appear the least times under the PSMM. This is due to the PSMM considering not only the one-step revenue of the sensor system but also the future expected revenue in decision-making, which can better predict the target state and thus select the nonblind sensor for tracking. The MSM only considers the one-step revenue in decision-making which is myopic for tracking, so the corresponding blind zone occurrence probability is higher than those of PSMM. The CSMM and FSMM do not select the sensor based on the tracking revenue in the future, which makes the blind zones occurred many times in the tracking.

Figures 9 and 10 show the comparisons of the RMSE and RTAMSE under different sensor management methods, respectively. Obviously, the PSMM can obtain the best tracking performance compared with the other methods. Besides, the position RMSE curves and velocity RMSE curves under PSMM are relatively stable in the simulation duration, and the corresponding error is basically not sharply increased, which means that it can achieve continuous and stable tracking of the target by the PSMM.

Figure 11 shows the sensor management schemes of the PSMM, which is the optimal solution of the objective function (47). It can be seen that the minimum continuous working time of the selected sensor satisfies the constraint that the minimum dwell time $t_{\min }=3 \mathrm{~s}$ in tracking, which verified that the management scheme is reasonable and effective.

Furthermore, in order to show that the proposed target state estimation algorithm based on the DBZ information is effective and advanced, the VSIMM-PF-DBZ algorithm is compared with the VSIMM-PF algorithm [43] in which the DBZ information is not be used. It can be seen from Figure $8(\mathrm{~d})$ that the DBZ will appear continuously during 4- $8 \mathrm{~s}$ under the FSMM. Therefore, we compare the two algorithms during 0-20 s under the FSMM in Figure 12, so as to study the tracking performance of them. Obviously, the tracking errors of the VSIMM-PF-DBZ are less than VSIMM-PF when the DBZ appears (4-8s), which indicates that the estimation algorithm proposed in this paper can reasonably use the DBZ information to track the target.

8.3. Analysis of the Proposed Motion Model. In this paper, a more realistic target motion model is established based on road topology information. To analyze the effect of utilizing road topology information in terms of tracking performance, the proposed motion model is compared with the model without utilizing any road topology information. Note that if no information is used, equation (2) is always used to describe the target motion in proposed tracking algorithm. Figure 13 shows the comparisons of the RMSE under different motion models. Obviously, the position and velocity RMSE under the proposed motion model are less than those under the motion model without road topology information. Especially when the target moves on the road $(0 \sim 45 \mathrm{~s}$ and 86 100s), the difference between the results with the two kinds of models is obvious. From the comparisons, we can see that the tracking accuracy is improved by utilizing road topology information to describe the target motion.

8.4. Analysis of the Proposed Decorrelation Method of the Measurement Noise. To analyze the performance of the proposed decorrelation method of the measurement noise, the results of tracking errors corresponding to using and not using decorrelation methods are compared. Note that the correlation coefficient $\omega$ is set as two values, 0.1 and 0.8 , and the other parameters remain unchanged.

Figures 14 and 15 show the comparison of the RMSE when $\omega=0.1$ and $\omega=0.8$, respectively. As can be seen, there is little difference between the results of the two methods when $\Phi=0.1$. On the contrary, when $\omega=0.8$, the results with decorrelation method are much better than those without decorrelation method. The comparison results show that the proposed decorrelation method is effective, especially when the correlation coefficient is large.

\section{Conclusions}

In this paper, a sensor management method for ground moving target tracking is proposed, in which the DBZ information is used to improve the tracking accuracy in the presence of the DBZ. Firstly, a more realistic motion model of the ground target is established, in which the target motion state is divided into two categories: off-road and on-road. Secondly, a sensor measurement model under measurement uncertainty is presented, and a decorrelation method is proposed to solve the problem that the measurement noises are statistically correlated. Third, a target state estimation algorithm based on the DBZ information is proposed, in which the DBZ information is fully utilized. Furthermore, combined with the VSIMM, an estimation algorithm is given to track the maneuvering target with multiple models. Finally, the PCRLB is used to quantify the tracking accuracy in the future, and a nonmyopic sensor management optimization model is established. Simulation results indicate that the proposed sensor management method can track the target accurately by selecting the suitable sensor management scheme at each time. Meanwhile, the proposed motion model and decorrelation method of the measurement noise are verified to be effective. As future work, we will study the sensor management method for multitarget tracking in the presence of the DBZ. 


\section{Data Availability}

The data used to support the findings of this study are available from the corresponding author upon request.

\section{Conflicts of Interest}

The authors declare that there are no conflicts of interest regarding the publication of this paper.

\section{Acknowledgments}

This research was funded by DPRFC (Defense Pre-Research Fund Project of China), grant number LJ20191C020393.

\section{References}

[1] M. Marcos, A. N. Vasconcelos, and M. Urbashi, "Optimal sensor management strategies in networked estimation," in Proceedings of IEEE 56th Annual Conference Decision and Control, pp. 5378-5385, Melbourne, VIC, Australia, December 2017.

[2] P. Salvagnini, F. Pernici, M. Cristani, G. Lisanti, A. Del Bimbo, and V. Murino, "Non-myopic information theoretic sensor management of a single pan-tilt-zoom camera for multiple object detection and tracking," Computer Vision and Image Understanding, vol. 134, pp. 74-88, 2015.

[3] H. G. Hoang and B. T. Vo, "Sensor management for multitarget tracking via multi-Bernoulli filtering," Automatica, vol. 50, no. 4, pp. 1135-1142, 2014.

[4] J. Z. HARE, S. GUPTA, and T. A. WETTERGREN, "POSE: prediction-based opportunistic sensing for energy efficiency in sensor networks using distributed supervisors," IEEE Transactions on Cybernetics, vol. 48, no. 7, pp. 2114-2127, 2018.

[5] J. Wu, Y. Zheng, Z. Sun, and H. Chen, "Multi-sensor management based on information increment and covariance of target priority discrimination," Telecommunications and Radio Engineering, vol. 79, no. 8, pp. 691-702, 2020.

[6] C. Pang and G. Shan, "Risk-based sensor scheduling for target detection," Computers \& Electrical Engineering, vol. 77, no. 7, pp. 179-190, 2019.

[7] A. S. Chhetri, D. Morrell, and A. Papandreou-Suppappola, "Efficient search strategies for non-myopic sensor scheduling in target tracking," in Conference Record of the Thirty-Eighth Asilomar Conference on Signals, Systems and Computers, 2004, pp. 2106-2110, Pacific Grove, CA, USA, November 2004.

[8] N. Cao, S. Choi, E. Masazade, and P. K. Varshney, "Sensor selection for target tracking in wireless sensor networks with uncertainty," IEEE Transactions on Signal Processing, vol. 64, no. 20, pp. 5191-5204, 2016.

[9] A. S. Chhetri, D. Morrell, and A. Papandreou-Suppappola, "On the use of binary programming for sensor scheduling," IEEE Transactions on Signal Processing, vol. 55, no. 6, pp. 2826-2839, 2007.

[10] X. S. Liu, L. Zhou, and X. Y. Du, "A method of sensor management based on target priority and information gain," Acta Electronica Sinica, vol. 33, no. 9, pp. 1683-1687, 2005.

[11] K. White, "Integrated tracking and sensor management based on expected information gain," in In Proceedings of SPIE-The International Society for Optical Engineering, Orlando, FL, USA, April 2007.
[12] X. Miao, J. Hu, G. Song, and A. Y. Zomaya, "Distributed segment-based anomaly detection with Kullback-Leibler divergence in wireless sensor networks," IEEE Transactions on Information Forensics and Security, vol. 12, no. 1, pp. 101-110, 2017.

[13] Q. Liu and Z. Liu, "A method of maneuvering target collaboration tracking based on Renyi information gain," Control and Decision, vol. 27, no. 9, pp. 1437-1440, 2012.

[14] C. M. Kreucher, A. O. Hero, and K. Kastella, "A comparison of task driven and information driven sensor management for target tracking," in Proceedings of the 44th IEEE Conference on Decision and Control, pp. 4004-4009, Seville, Spain, December 2005.

[15] F. Katsilieris, H. Driessen, and A. Yarovoy, "Threat-based sensor management for target tracking," IEEE Transactions on Aerospace and Electronic Systems, vol. 51, no. 4, pp. 27722785, 2015.

[16] C. Pang, G. Shan, X. Duan, and G. Xu, “A multi-mode sensor management approach in the missions of target detecting and tracking," Electronics, vol. 8, no. 1, p. 71, 2019.

[17] S. Martin, "Risk-based sensor resource management for field of view constrained sensors," in 2015 18th International Conference on Information Fusion (Fusion), 2048, p. 2041, Washington, DC, USA, July 2015.

[18] C. Shi, W. Fei, S. Mathini, and J. Zhou, "LPI optimization framework for target tracking in radar network architectures using information-theoretic criteria," International Journal of Antennas \& Propagation, vol. 2014, pp. 1-10, 2014.

[19] Z. N. Zhang and G. L. Shan, "UTS-based foresight optimization of sensor scheduling for low interception risk tracking," International Journal of Adaptive Control \& Signal Processing, vol. 28, no. 10, pp. 921-931, 2014.

[20] V. Krishnamurthy, "Emission management for low probability intercept sensors in network centric warfare," IEEE Transactions on Aerospace and Electronic Systems, vol. 41, no. 1, pp. 133-152, 2005.

[21] M. Kalandros and L. Pao, "Multisensor covariance control strategies for reducing bias effects in interacting target scenarios," IEEE Transactions on Aerospace and Electronic Systems, vol. 41, no. 1, pp. 153-173, 2005.

[22] C. Yang, L. Kaplan, and E. Blasch, "Performance measures of covariance and information matrices in resource management for target state estimation," IEEE Transactions on Aerospace and Electronic Systems, vol. 48, no. 3, pp. 2594-2613, 2012.

[23] J. M. Dias and P. A. Marques, "Multiple moving target detection and trajectory estimation using a single SAR sensor," IEEE Transactions on Aerospace and Electronic Systems, vol. 39, no. 2, pp. 604-624, 2003.

[24] G. Xu, G. Shan, and X. Duan, "Non-myopic scheduling method of mobile sensors for manoeuvring target tracking," IET Radar, Sonar \& Navigation, vol. 13, no. 11, pp. 18991908, 2019.

[25] A. Mohammadi and A. Asif, "Consensus-based distributed dynamic sensor selection in decentralised sensor networks using the posterior Cramer-Rao lower bound," Signal Processing, vol. 108, pp. 558-575, 2015.

[26] A. K. Gostar, R. Hoseinnezhad, T. Rathnayake, X. Wang, and A. Bab-Hadiashar, "Constrained sensor control for labeled multi-Bernoulli filter using Cauchy-Schwarz divergence," IEEE Signal Processing Letters, vol. 24, no. 9, pp. 1313-1317, 2017. 
[27] K. Wan, X. Gao, L. I. Bo, and L. I. Fei, "Using approximate dynamic programming for multi-ESM scheduling to track ground moving targets," Journal of Systems Engineering \& Electronics, vol. 29, no. 1, pp. 74-85, 2018.

[28] Y. Cheng and T. Singh, "Efficient particle filtering for roadconstrained target tracking," IEEE Transactions on Aerospace \& Electronic Systems, vol. 43, no. 99, pp. 1454-1469, 2007.

[29] Y. Miao, C. Liu, W. H. Chen, and J. Chambers, "A Bayesian framework with an auxiliary particle filter for GMTI-based ground vehicle tracking aided by domain knowledge," in In Proceedings of SPIE-The International Society for Optical Engineering, Baltimore, Maryland, United States, June 2014.

[30] M. Ulmke and W. Koch, "Road-map assisted ground moving target tracking," IEEE Transactions on Aerospace and Electronic Systems, vol. 42, no. 4, pp. 1264-1274, 2006.

[31] W. Koch, J. Koller, and M. Ulmke, "Ground target tracking and road map extraction," Isprs Journal of Photogrammetry \& Remote Sensing, vol. 61, no. 3-4, pp. 197-208, 2006.

[32] Hyondong Oh, Seungkeun Kim, and A. Tsourdos, "Roadmap-assisted standoff tracking of moving ground vehicle using nonlinear model predictive control," IEEE Transactions on Aerospace \& Electronic Systems, vol. 51, no. 2, pp. 975-986, 2015.

[33] Z. Xinyan and Z. Wei, "Application of road information in ground moving target tracking," Chinese Journal of Aeronautics, vol. 20, no. 6, pp. 529-538, 2007.

[34] J. Zheng and M. Gao, "Tracking ground targets with a road constraint using a GMPHD filter," Sensors, vol. 18, no. 8, pp. 2723-2743, 2018.

[35] M. Arulampalam, N. Gordon, M. Orton, and B. Ristic, "A variable structure multiple model particle filter for GMTI tracking," in Proceedings of the 15th International Conference on Information Fusion, pp. 927-934, Annapolis, MD, USA, July 2002.

[36] X. Gongguo, S. Ganlin, and D. Xiusheng, "Sensor scheduling for ground maneuvering target tracking in presence of detection blind zone," Journal of Systems Engineering and Electronics, vol. 31, no. 4, pp. 692-702, 2020.

[37] W. Wu, W. Liu, J. Jiang, L. Gao, Q. Wei, and C. Liu, "GM-PHD filter-based multi-target tracking in the presence of Doppler blind zone," Digital Signal Processing, vol. 52, pp. 1-12, 2016.

[38] W. Wu, H. Sun, Y. Cai, and J. Xiong, "MM-GLMB filter-based sensor control for tracking multiple maneuvering targets hidden in the Doppler blind zone," IEEE Transactions on Signal Processing, vol. 68, pp. 4555-4567, 2020.

[39] H. Huang, R. Yang, P. Foo et al., "Convoy tracking in Doppler blind zone regions using GMTI radar," in Proceedings of the 16th International Conference on Information Fusion, pp. 1768-1775, Istanbul, Turkey, July 2013.

[40] M. Mertens and U. Nickel, "GMTI tracking in the presence of Doppler and range ambiguities," in Proceedings of the 14th International Conference on Information Fusion, pp. 13691376, Chicago, Illinois, USA, July 2011.

[41] M. Lei and C. Han, "Sequential nonlinear tracking using UKF and raw range-rate measurements," IEEE Transactions on Aerospace \& Electronic Systems, vol. 43, no. 1, pp. 239-250, 2007.

[42] M. Byrne, K. White, and J. Williams, "Rolling horizon nonmyopic scheduling of multifunction radar for search and track," in International Conference on Information Fusion, pp. 634-642, Heidelberg, Germany, July 2016.
[43] M. Yu, H. Oh, and W. Chen, "An improved multiple model particle filtering approach for manoeuvring target tracking using airborne GMTI with geographic information," Aerospace Science and Technology, vol. 52, no. 5, pp. 62-69, 2016.

[44] B. R. Mahafza, Radar Systems Analysis and Design Using $M A T L A B$, Publishing House Electronic Industry, Beijing, China, 2008.

[45] C. Gao and W. Chen, "Ground moving target tracking with VS-IMM using mean shift unscented particle filter," Chinese Journal of Aeronautics, vol. 24, no. 5, pp. 622-630, 2011. 